\title{
X-RAY EMISSION LINE SPECTROSCOPY OF CATACLYSMIC VARIABLES. II. TEMPERATURES AND DENSITIES FROM LINE RATIOS IN THE CHANDRA HETG BAND
}

\author{
E. M. Schlegel ${ }^{1,6}$, H. V. Shipley ${ }^{1,2}$, V. R. Rana ${ }^{3}$, P. E. BarretT ${ }^{4}$, And K. P. Singh ${ }^{5}$ \\ ${ }^{1}$ Department of Physics and Astronomy, University of Texas-San Antonio, San Antonio, TX 78249, USA; eric.schlegel@utsa.edu \\ 2 Texas A\&M University, College Station, TX, USA \\ 3 Space Radiation Laboratory, Caltech, Pasadena, CA 91125, USA; vrana@ srl.caltech.edu \\ ${ }^{4}$ US Naval Observatory, Washington, DC 20392-5420, USA; barrett.paul@usno.navy.mil \\ 5 Tata Institute of Fundamental Research, Homi Bhabha Road, Mumbai, India; singh@tifr.res.in \\ Received 2012 May 29; accepted 2013 March 26; published 2014 November 24
}

\begin{abstract}
We summarize the results of a line-by-line fitting analysis of the available spectra obtained using the Chandra High-Energy Transmission Grating. We confirm the existence of broad ionization and electron temperature ranges and high number densities in cataclysmic variables (CVs) of all subtypes. Temperatures range from $\sim 0.4 \mathrm{keV}$ to $\sim 5-10 \mathrm{keV}$ or more with a broad range detected in any given CV. In other words, single-temperature models do not describe the line emission. Number densities also cover a broad range, from $10^{12}$ to $>10^{16} \mathrm{~cm}^{-3}$. We demonstrate that much of the plasma is in a nonequilibrium state; the Fe emission, however, may arise from plasma in the ionization equilibrium.
\end{abstract}

Key words: novae, cataclysmic variables - stars: dwarf novae - X-rays: binaries

Online-only material: color figures

\section{INTRODUCTION}

$\mathrm{X}$-ray emission from cataclysmic variables (CVs) is important because of its potential to probe the accretion process in a direct manner. The emission originates from varying locations depending upon the CV subclass. For magnetic systems (polars), $\mathrm{X}$-rays originate from in, behind, or near the accretion shock formed as the incoming mass transfer stream settles onto the white dwarf (WD; Aizu 1973; Cropper 1990; Warner 1995). Hard X-rays arise from the shock itself while soft X-rays are expected to originate from hard X-rays that have been absorbed by the WD surface and re-radiated at lower energies. A similar picture occurs in the intermediate polars (IPs) except the accretion structure is thought to resemble an arc or curtain instead of a stream or funnel (Cropper 1986; Rosen et al. 1988). For dwarf novae, novalikes, and old novae, the production of $\mathrm{X}$-rays is expected to occur by the dissipation of accretion energy in the boundary layer (BL; Warner 1995).

Low-resolution (CCD) spectra have been extensively studied. For example, Ezuka \& Ishida (1999) analyzed 23 ASCA spectra of magnetic CVs and IPs, fitting the data with multiple emission and absorption components. E. M. Schlegel (2000, unpublished) showed that the spectra were also statistically consistent with simpler models. The low resolution of the data complicated the analysis given the multiple line identifications possible, particularly in the Fe L band between $\sim 10$ and $17 \AA(0.7-1 \mathrm{keV})$ where a broad range of ionization states are found ( $\sim \mathrm{Fe}$ XVII to Fe XXIII).

Dispersive spectroscopy at resolutions of $\Delta E / E \sim 0.01$ in the X-ray band were first available in the late 1960s through the mid-1970s on various instruments (see Lum et al. 1992, and references therein). Given the small effective areas of the instruments, the use of nonimaging optics, and the typically low X-ray photon event rates of most astrophysical objects, relatively few targets were available for study. The Einstein Focal Plane Crystal Spectrometer (FPCS) was essentially the

\footnotetext{
6 Vaughan Family Professor.
}

first productive instrument for a large number of nonsolar observations. A summary by Lum et al. (1992) of the Einstein spectrometer lists 41 sources including 2 CVs (AM Her and SS Cyg). Neither CV was detected at the exposure times. While appropriate for a first-time survey, these detections took place at a small fraction of the time required to deliver spectra with good signal-to-noise (exposure times $\sim 5-10 \mathrm{ks}$ ). Other $\mathrm{X}$-ray detectors that followed Einstein (alphabetically: ASCA, BBXRT, EXOSAT, Ginga, ROSAT, RXTE) all carried detectors with spectral resolutions $\sim 5-10$ times lower than the FPCS.

The launches of Chandra and XMM-Newton, each carrying a set of gratings (the High-Energy Transmission Grating (HETG) and Low-Energy Transmission Grating on Chandra and the Reflection Grating Spectrometer on XMM-Newton), allowed for the study of grating spectra of not only broad classes of objects but also significant numbers within each class. The detected spectra occur in sufficient numbers and with sufficiently high signal-to-noise to initiate the investigation of systematic behaviors of representatives of X-ray-emitting classes of objects.

$\mathrm{X}$-ray spectroscopy from high-resolution gratings provides the potential for the direct investigation of accretion physics, particularly in CVs where their relative proximity reduces the likelihood of high column densities toward the objects of study. Analyses of the lines and various line ratios can, in principle, provide the measurements of temperatures, densities, opacity effects, and emission geometries necessary to understand the physics and to model the details of the accretion process in its manifestations among the subclasses of CVs. The 1.5-25 $(\sim 0.5-8.3 \mathrm{keV})$ band is one of the best spectral regions for such investigations because of the presence of a large number of spectral lines from a variety of ionization states and ions.

The motivation for the analysis approach in this study started with the publication of Mukai et al. (2003) in which the authors fit CV HETG spectra with "global" models. They argued that the seven spectra then available fell into two broad groups, the "cooling flow" spectra, best described by a cooling, optically thin thermal gas model, and the "photoionized" spectra. While we do not necessarily disagree with their conclusions, we noted 
Table 1

List of HETG Observations

\begin{tabular}{|c|c|c|c|c|c|}
\hline $\mathrm{CV}$ & $\begin{array}{l}\text { CV } \\
\text { Type }\end{array}$ & ObsId & ObsDate & $\begin{array}{c}\text { ExposT } \\
(\mathrm{ks})\end{array}$ & State $^{\mathrm{a}}$ \\
\hline V603 Aql & NX & 1901 & 2001 Apr 19 & 65 & Quiescent \\
\hline AE Aqr & $\mathrm{AM}$ & 5431 & 2005 Aug 30 & 78 & $\cdots$ \\
\hline \multirow[t]{4}{*}{ TT Ari } & $\cdots$ & 6372 & 2005 Sep 6 & 33 & $\cdots$ \\
\hline & & 5432 & 2005 Oct 4 & 24 & $\cdots$ \\
\hline & & 7176 & 2005 Oct 6 & 24 & $\cdots$ \\
\hline & & 7175 & 2005 Oct 9 & 15 & $\cdots$ \\
\hline V834 Cen & $\mathrm{AM}$ & 3768 & 2003 Sep 13 & 60 & $\cdots$ \\
\hline \multirow[t]{3}{*}{ SS Cyg } & DN & 646 & 2000 Aug 24 & 48 & Quiescent \\
\hline & & 2307 & 2000 Sep 12 & 37 & Outburst (1) \\
\hline & & 648 & 2000 Sep 14 & 60 & Outburst (2) \\
\hline \multirow[t]{3}{*}{ YY Dra } & IP & 5433 & 2006 Jan 18 & 32 & $\cdots$ \\
\hline & & 7261 & 2006 Jan 20 & 45 & $\ldots$ \\
\hline & & 7262 & 2006 Jan 21 & 23 & $\cdots$ \\
\hline \multirow[t]{2}{*}{ U Gem } & DN & 647 & 2000 Nov 29 & 96 & Quiescent \\
\hline & & 3767 & 2002 Dec 26 & 62 & Outburst \\
\hline AM Her & $\mathrm{AM}$ & 3769 & 2003 Aug 15 & 93 & $\ldots$ \\
\hline \multirow[t]{5}{*}{ EX Нуа } & IP & 1706 & 2000 May 18 & 60 & $\cdots$ \\
\hline & & 7449 & 2007 May 13 & 131 & $\cdots$ \\
\hline & & 7452 & 2007 May 17 & 49 & $\cdots$ \\
\hline & & 7450 & 2007 May 18 & 163 & $\ldots$ \\
\hline & & 7451 & 2007 May 21 & 153 & $\cdots$ \\
\hline \multirow[t]{2}{*}{ WX Hyi } & DN & 2670 & 2002 Jul 25 & 50 & Quiescent \\
\hline & & 3721 & 2002 Jul 28 & 49 & Quiescent \\
\hline V426 Oph & DN & 2671 & 2002 May 30 & 45 & Quiescent \\
\hline \multirow[t]{2}{*}{ GK Per } & IP & 3454 & 2002 Mar 27 & 32 & Outburst (1) \\
\hline & & 3455 & 2002 Apr 9 & 34 & Outburst (2) \\
\hline AO Psc & IP & 1898 & 2001 May 23 & 100 & $\cdots$ \\
\hline V1223 Sgr & IP & 649 & 2000 Apr 30 & 52 & $\cdots$ \\
\hline \multirow[t]{2}{*}{ SU UMa } & DN & 3478 & 2002 Apr 12 & 24 & Quiescent \\
\hline & & 2680 & 2002 Apr 13 & 50 & Quiescent \\
\hline
\end{tabular}

Note. ${ }^{a}$ The number for CVs with multiple observations provides the link to subsequent tables.

that their modeled lines in many cases over- or under-predicted line strengths relative to the observed line emission. We became interested in what physics could be inferred based on a "local" analysis of the spectra. We were also aware of an increasing number of model predictions of line ratios and wanted to make a comparison between the observations and the predictions.

This paper focuses on the ratios of lines detected in the spectra of CVs obtained using the HETG on Chandra (Canizares et al. 2005). The HETG consists of two gratings: the High-Energy Grating (HEG) and the Medium-Energy Grating (MEG). Our analysis covers both data sets and the description of the analysis is detailed in (Shipley et al. 2015; Paper I of the series). A sample of plots is presented in the physical paper; the remaining plots are available in the online version.

\section{OBSERVATIONS AND ANALYSIS}

A total of 30 observations of $15 \mathrm{CVs}$ have been collected using the HETG as listed in Table 1 . We briefly summarize the reduction of the data and refer the reader to Shipley et al. (2015) for additional details.

The data, extracted from the Chandra archive, were reprocessed during 2006-2008 in an effort by the Chandra data processing team to bring the archive to a uniform processing level. Significant changes in the standard processing include corrections for time-dependent gain, charge transfer inefficiency (including time-dependence in the charge transfer inefficiency), and time-dependent absorption on the detector entrance window.
The standard grating spectroscopy processing "thread" was followed. ${ }^{7}$ The data were extracted and subsequently binned to a uniform wavelength scale using $0.005 \AA$ bins. An effective area curve was generated for each observation and was rebinned to the same scale. The counts in each bin were corrected for the wavelength-dependent area. As described in Shipley et al. (2015), HEG data were used for Fe and S lines; MEG data was used for all other lines.

Our primary goal for line fitting was a robust measure of the emission line flux. To fit the overall continuum, the emission lines were first blocked and the remaining channels were fit with low-order terms to obtain a smooth function through the continuum. Each line was modeled using a loworder polynomial fit to the local continuum plus one or more Gaussians using a Bayesian line fitting routine (Sivia 2006).

Each line's Gaussian width was tested for a significant nonzero value, but, in general, the data were consistent with unresolved lines with the exception of lines of the CVs observed in the outburst as first noted by Mauche et al. (2003b). Hence, the Gaussian widths were fixed at zero to mimic unresolved lines except for the outburst spectra. The Gaussian centers were fixed at the center of each individual emission line. Once a good fit was obtained, the line center was permitted to vary to test for nonzero velocity offsets. The line center was then refixed at the best-fit value and the $90 \%$ error on the line flux was determined. Errors were determined from the Hessian matrix as outlined in Sivia (2006).

Upper limits were determined by fixing a zero-width Gaussian at the expected line position and increasing the flux until $\Delta \chi^{2}$ increased by 2.71 , corresponding to the $90 \%$ increase for 1 degree of freedom.

Ratios were then calculated for the H-like and He-like resonance lines (Table 2), the triplet lines contributing to the $G$ (Table 3 ) and $R$ (Table 4 ) ratios, and the ratios of the various lines of $\mathrm{Fe}$ of intermediate ionization (Table 5).

For a direct comparison of observations and models, we employed the mkcflow model described in Mukai et al. (2003). We used that model with the updated atomic physics values currently available in the Astrophysical Plasma Emission Database (Smith et al. 2001). Mukai et al. (2003) noted that the CV spectra suggested a flat emission measure distribution in a multitemperature plasma. Mukai et al. fit the overall spectrum using the cooling flow model, demonstrating that the derived cooling flow rates were in line with estimates for mass accretion rates from other analyses. Here we generate a series of models varying the primary model parameter $T_{\max }$, the maximum temperature of the plasma, and fit the resulting lines for comparison with the observed line emission.

We also used the photoionization code XSTAR to produce several models for comparison with the observations (Kallman 1999). XSTAR contains a large number of parameters-we focused on narrow ranges for just a few illustrative parameters ${ }^{8}$ : gas temperature $\left(10^{5}, 10^{6}, 2 \times 10^{6}\right.$ and $\left.4 \times 10^{6} \mathrm{~K}\right)$, the temperature of the radiation $(15,20$, and $25 \mathrm{keV})$, gas density $\left(10^{14}\right.$, $10^{15}$, and $\left.10^{16} \mathrm{~cm}^{-3}\right)$, and the local column density $\left(10^{17}, 10^{19}\right.$, $10^{21}$, and $10^{23} \mathrm{~cm}^{-2}$ ). We found that the radiation temperature altered the line ratios minimally over the adopted range. The largest impact occurred at high radiation temperatures and low gas densities.

\footnotetext{
7 Analysis and science threads are available at http://cxc.harvard.edu/ciao. 8 Given the large errors, a more complete exploration of model parameters is not justified because models with many different parameters would lead to similar line ratios.
} 
Table 2

Ratios for $\mathrm{H}$-like $\mathrm{K} \alpha / \mathrm{He}$-like Resonance

\begin{tabular}{lcccccc}
\hline \hline CV & Fe XXVI $\alpha /$ Fe XXV-r & S XVI $\alpha /$ S xV-r & Si XIV $\alpha /$ Si XIII-r & Mg XII $\alpha /$ Mg XI-r & Ne x $\alpha /$ Ne IX-r & O vIII $\alpha / O$ VII-r \\
\hline V603 Aql & $0.46 \pm 0.30$ & $<2.89$ & $2.06 \pm 0.81$ & $3.18 \pm 1.45$ & $2.41 \pm 1.07$ & $4.72 \pm 2.52$ \\
AE Aqr & $<0.65$ & $<1.20$ & $2.06 \pm 0.48$ & $1.12 \pm 0.70$ & $1.56 \pm 0.31$ & $10.13 \pm 9.76$ \\
TT Ari & $0.07 \pm 0.04$ & $3.52 \pm 1.99$ & $1.68 \pm 0.37$ & $2.12 \pm 0.99$ & $1.50 \pm 0.59$ & $<2.56$ \\
V834 Cen & $0.98 \pm 0.70$ & $14.00 \pm 9.75$ & $1.95 \pm 0.85$ & $2.34 \pm 1.53$ & $1.82 \pm 1.09$ & $1.49 \pm 1.05$ \\
SS Cyg-Q & $1.05 \pm 0.52$ & $2.03 \pm 0.83$ & $18.67 \pm 12.44$ & $5.68 \pm 3.49$ & $2.19 \pm 0.42$ & $2.11 \pm 1.71$ \\
SS Cyg-O1 & $0.90 \pm 0.49$ & $<0.92$ & $1.15 \pm 0.22$ & $4.32 \pm 3.74$ & $1.28 \pm 0.39$ & $0.26 \pm 0.16$ \\
SS Cyg-O2 & $<0.50$ & $2.94 \pm 1.84$ & $1.57 \pm 0.28$ & $2.91 \pm 0.81$ & $0.63 \pm 0.49$ & $0.34 \pm 0.20$ \\
YY Dra & $1.62 \pm 1.08$ & $0.66 \pm 0.29$ & $5.76 \pm 3.91$ & $5.69 \pm 3.56$ & $4.68 \pm 2.69$ & $4.38 \pm 3.18$ \\
UG Gem-Q & $0.64 \pm 0.29$ & $3.71 \pm 3.56$ & $3.79 \pm 1.57$ & $3.21 \pm 1.42$ & $3.69 \pm 2.01$ & $<9.32$ \\
UG Gem-O & $3.17 \pm 2.46$ & $1.40 \pm 1.29$ & $10.10 \pm 4.83$ & $1.86 \pm 1.05$ & $1.68 \pm 0.42$ & $2.62 \pm 1.59$ \\
AM Her & $0.51 \pm 0.10$ & $9.34 \pm 8.30$ & $7.62 \pm 3.66$ & $9.61 \pm 6.13$ & $3.67 \pm 1.50$ & $0.31 \pm 0.14$ \\
EX Hya2000 & $0.37 \pm 0.16$ & $2.64 \pm 1.35$ & $2.61 \pm 0.34$ & $2.01 \pm 0.23$ & $3.12 \pm 0.54$ & $9.69 \pm 7.28$ \\
EX Hya2007 & $0.59 \pm 0.07$ & $1.93 \pm 0.80$ & $2.31 \pm 0.22$ & $2.71 \pm 0.43$ & $4.03 \pm 1.00$ & $7.24 \pm 0.98$ \\
WX Hyi & $0.21 \pm 0.12$ & $1.87 \pm 1.60$ & $3.23 \pm 0.96$ & $3.32 \pm 1.80$ & $5.80 \pm 3.02$ & $2.57 \pm 1.60$ \\
V426 Oph & $0.77 \pm 0.27$ & $5.82 \pm 3.92$ & $1.99 \pm 0.53$ & $6.20 \pm 5.03$ & $2.03 \pm 1.60$ & $<0.73$ \\
GK Per-1 & $3.99 \pm 3.22$ & $1.59 \pm 1.44$ & $<0.73$ & $0.35 \pm 0.25$ & $0.51 \pm 0.44$ & $3.06 \pm 2.62$ \\
GK Per-2 & $2.47 \pm 1.67$ & $1.16 \pm 1.06$ & $0.25 \pm 0.20$ & $0.23 \pm 0.19$ & $0.32 \pm 0.25$ & $0.69 \pm 0.68$ \\
GK Per-Av & $1.37 \pm 0.87$ & $<0.91$ & $0.34 \pm 0.12$ & $0.97 \pm 0.24$ & $0.61 \pm 0.45$ & $1.21 \pm 0.57$ \\
AO Psc & $0.60 \pm 0.43$ & $1.68 \pm 1.44$ & $<4.24$ & $3.92 \pm 2.50$ & $<4.49$ & $<0.64$ \\
V1223 Sgr & $2.88 \pm 1.61$ & $0.93 \pm 0.85$ & $11.69 \pm 9.30$ & $2.59 \pm 1.51$ & $2.99 \pm 1.85$ & $0.92 \pm 0.50$ \\
SU UMa & $0.56 \pm 0.27$ & $<6.13$ & $1.81 \pm 0.67$ & $1.75 \pm 1.46$ & $5.34 \pm 2.85$ & $<4.98$ \\
\hline
\end{tabular}

Table 3

$G$ Ratios for He-like Triplets

\begin{tabular}{|c|c|c|c|c|c|c|}
\hline$\overline{\mathrm{CV}}$ & Fexxv & $\mathrm{Sxv}$ & Si XIII & Mg XI & Ne IX & O VII \\
\hline V603 Aql & $0.34 \pm 0.20$ & $0.65 \pm 0.57$ & $0.68 \pm 0.35$ & $1.22 \pm 0.86$ & $0.81 \pm 0.45$ & $1.29 \pm 0.67$ \\
\hline AE Aqr & $<1.06$ & $2.84 \pm 2.79$ & $0.64 \pm 0.31$ & $<0.85$ & $0.25 \pm 0.09$ & $<1.21$ \\
\hline TT Ari & $1.10 \pm 0.63$ & $3.70 \pm 2.13$ & $0.61 \pm 0.19$ & $1.14 \pm 0.77$ & $0.46 \pm 0.32$ & $<2.86$ \\
\hline V834 Cen & $0.73 \pm 0.52$ & $6.00 \pm 4.31$ & $0.90 \pm 0.46$ & $1.37 \pm 0.91$ & $1.15 \pm 0.78$ & $2.47 \pm 1.25$ \\
\hline SS Cyg-Q & $1.06 \pm 0.46$ & $1.37 \pm 0.60$ & $2.23 \pm 1.96$ & $1.00 \pm 0.84$ & $0.30 \pm 0.13$ & $1.12 \pm 1.01$ \\
\hline SS Cyg-O1 & $3.40 \pm 1.83$ & $2.16 \pm 2.00$ & $0.39 \pm 0.22$ & $0.32 \pm 0.30$ & $0.45 \pm 0.16$ & $0.58 \pm 0.22$ \\
\hline SS Cyg-O2 & $4.19 \pm 1.72$ & $2.22 \pm 1.35$ & $0.64 \pm 0.30$ & $1.43 \pm 0.44$ & $0.80 \pm 0.16$ & $1.18 \pm 0.34$ \\
\hline YY Dra & $1.51 \pm 1.02$ & $3.17 \pm 0.99$ & $0.86 \pm 0.58$ & $2.15 \pm 1.49$ & $1.31 \pm 0.84$ & $2.91 \pm 1.66$ \\
\hline UG Gem-Q & $0.69 \pm 0.27$ & $<2.48$ & $0.83 \pm 0.50$ & $0.44 \pm 0.36$ & $0.82 \pm 0.65$ & $<2.67$ \\
\hline UG Gem-O & $13.67 \pm 8.95$ & $1.96 \pm 1.39$ & $4.97 \pm 2.46$ & $0.47 \pm 0.33$ & $0.31 \pm 0.16$ & $1.97 \pm 1.32$ \\
\hline AM Her & $0.19 \pm 0.11$ & $2.95 \pm 2.12$ & $2.30 \pm 1.82$ & $2.48 \pm 2.21$ & $1.35 \pm 0.68$ & $0.63 \pm 0.27$ \\
\hline EX Hya2000 & $0.12 \pm 0.04$ & $0.73 \pm 0.44$ & $0.71 \pm 0.17$ & $0.36 \pm 0.13$ & $0.68 \pm 0.25$ & $0.98 \pm 0.91$ \\
\hline EX Нya2007 & $0.58 \pm 0.07$ & $0.57 \pm 0.26$ & $0.55 \pm 0.05$ & $0.65 \pm 0.13$ & $0.67 \pm 0.17$ & $0.79 \pm 0.15$ \\
\hline WX Hyi & $0.87 \pm 0.35$ & $0.97 \pm 0.70$ & $0.46 \pm 0.19$ & $0.81 \pm 0.44$ & $0.14 \pm 0.09$ & $1.37 \pm 0.61$ \\
\hline V426 Oph & $0.19 \pm 0.08$ & $2.38 \pm 1.84$ & $0.54 \pm 0.43$ & $1.55 \pm 1.21$ & $0.60 \pm 0.48$ & $<2.21$ \\
\hline GK Per-1 & $11.33 \pm 7.50$ & $<2.80$ & $1.92 \pm 1.24$ & $2.16 \pm 1.06$ & $1.55 \pm 1.02$ & $1.56 \pm 1.26$ \\
\hline GK Per-2 & $0.84 \pm 0.66$ & $1.86 \pm 1.54$ & $4.16 \pm 2.39$ & $1.00 \pm 0.64$ & $1.08 \pm 0.70$ & $0.62 \pm 0.60$ \\
\hline GK Per-Av & $1.30 \pm 0.98$ & $<1.92$ & $2.12 \pm 0.52$ & $2.29 \pm 0.47$ & $1.41 \pm 0.78$ & $0.60 \pm 0.48$ \\
\hline AO Psc & $0.57 \pm 0.19$ & $2.15 \pm 1.92$ & $<1.60$ & $1.02 \pm 0.80$ & $<3.42$ & $<1.19$ \\
\hline V1223 Sgr & $0.86 \pm 0.45$ & $1.89 \pm 1.31$ & $6.05 \pm 4.88$ & $2.59 \pm 1.41$ & $2.01 \pm 1.34$ & $0.38 \pm 0.23$ \\
\hline SU UMa & $1.24 \pm 0.80$ & $6.08 \pm 4.82$ & $0.78 \pm 0.33$ & $0.30 \pm 0.22$ & $1.24 \pm 0.71$ & $3.82 \pm 2.63$ \\
\hline
\end{tabular}

We also refer the reader to the discussion in Perna et al. (2003) for additional model possibilities including hot settling flow, coronal siphon flow, or X-ray emitting corona. However, none of those models provided a good match to the observed spectra.

Finally, we include for comparison, when appropriate, the values for several line ratios from the XMM-Newton observation of GK Per (Vrielmann et al. 2005) and the HETG observation of Capella (Behar et al. 2001; Canizares et al. 2000, 2005). All data points refer to the key shown in Figure 1 (Shipley et al. 2015).

\section{LINE RATIOS}

Figure 1 shows the key used throughout the paper. Magnetic $\mathrm{CVs}$ are displayed as black points while nonmagnetic CVs are displayed as red. Shapes are used to distinguish individual CVs, but many of the shapes are used twice: for a magnetic and a nonmagnetic CV. Data points without errors represent the $90 \%$ upper limit on the ratio for that CV.

In all of the figures presenting line ratios, the data points are placed at an arbitrary independent parameter value (e.g., temperature or density) so that individual CVs may be identified. This is done solely to separate the points horizontally; in many cases, were this not done, the data points would lie on top of each other.

The interpretation of the figure then depends upon where the data points fall relative to the curve: given that the data have been arbitrarily positioned horizontally for visibility, each data point may be moved horizontally until it encounters the curve. For example, for the magnetic CVs in the $\mathrm{H}$-like/He-like $\mathrm{K} \alpha$ (Figure 2(a)), the third data point from the left translates to an 
Table 4

$R$ Ratios for He-like Triplets

\begin{tabular}{|c|c|c|c|c|c|c|}
\hline $\mathrm{CV}$ & Fe XXv & S XV & Si XIII & Mg XI & Ne IX & O VII \\
\hline V603 Aql & $0.84 \pm 0.69$ & $<0.94$ & $0.90 \pm 0.74$ & $0.64 \pm 0.61$ & $0.21 \pm 0.14$ & $<0.97$ \\
\hline AE Aqr & $<0.08$ & $<3.66$ & $1.12 \pm 0.98$ & $<4.94$ & $4.40 \pm 3.78$ & $<1.14$ \\
\hline TT Ari & $0.10 \pm 0.08$ & $<0.71$ & $0.95 \pm 0.50$ & $0.34 \pm 0.29$ & $0.27 \pm 0.25$ & $<0.29$ \\
\hline V834 Cen & $0.23 \pm 0.20$ & $0.46 \pm 0.39$ & $0.40 \pm 0.29$ & $0.56 \pm 0.42$ & $1.05 \pm 0.92$ & $<0.95$ \\
\hline SS Cyg-Q & $<0.48$ & $0.86 \pm 0.45$ & $<1.36$ & $<1.17$ & $1.24 \pm 1.00$ & $0.25 \pm 0.22$ \\
\hline SS Cyg-O1 & $0.43 \pm 0.28$ & $2.20 \pm 1.89$ & $0.45 \pm 0.40$ & $1.09 \pm 0.91$ & $0.27 \pm 0.17$ & $0.21 \pm 0.15$ \\
\hline YY Dra & $0.51 \pm 0.43$ & $0.83 \pm 0.39$ & $1.29 \pm 0.66$ & $0.50 \pm 0.38$ & $0.41 \pm 0.29$ & $<0.74$ \\
\hline UG Gem-Q & $0.18 \pm 0.14$ & $<1.12$ & $<0.19$ & $<0.91$ & $<0.63$ & $<0.62$ \\
\hline UG Gem-O & $0.07 \pm 0.05$ & $0.79 \pm 0.59$ & $0.26 \pm 0.07$ & $1.20 \pm 1.13$ & $0.25 \pm 0.21$ & $<0.08$ \\
\hline AM Her & $<0.06$ & $0.77 \pm 0.58$ & $<2.56$ & $<0.77$ & $0.62 \pm 0.46$ & $0.19 \pm 0.16$ \\
\hline EX Hya2000 & $0.06 \pm 0.05$ & $0.86 \pm 0.70$ & $0.39 \pm 0.23$ & $0.14 \pm 0.12$ & $0.27 \pm 0.15$ & $<0.05$ \\
\hline EX Hya2007 & $0.09 \pm 0.01$ & $0.53 \pm 0.19$ & $0.31 \pm 0.05$ & $0.09 \pm 0.02$ & $0.05 \pm 0.02$ & $<0.06$ \\
\hline WX Hyi & $0.06 \pm 0.04$ & $1.04 \pm 0.93$ & $1.13 \pm 0.79$ & $0.62 \pm 0.25$ & $0.74 \pm 0.61$ & $<0.28$ \\
\hline GK Per-1 & $0.11 \pm 0.08$ & $<0.15$ & $<2.07$ & $0.64 \pm 0.41$ & $1.02 \pm 0.83$ & $1.88 \pm 1.68$ \\
\hline GK Per-2 & $0.14 \pm 0.12$ & $<1.33$ & $0.98 \pm 0.43$ & $<0.40$ & $0.61 \pm 0.39$ & $<0.05$ \\
\hline GK Per-Av & $<0.06$ & $<0.72$ & $3.50 \pm 1.66$ & $0.92 \pm 0.28$ & $0.66 \pm 0.48$ & $<0.17$ \\
\hline AO Psc & $0.04 \pm 0.03$ & $2.77 \pm 2.69$ & $<0.47$ & $<0.77$ & $<0.74$ & $<0.47$ \\
\hline V1223 Sgr & $0.10 \pm 0.08$ & $0.29 \pm 0.20$ & $0.13 \pm 0.07$ & $0.20 \pm 0.13$ & $0.21 \pm 0.19$ & $0.26 \pm 0.21$ \\
\hline SU UMa & $0.27 \pm 0.20$ & $<0.86$ & $0.50 \pm 0.38$ & $<1.58$ & $0.86 \pm 0.58$ & $<0.32$ \\
\hline
\end{tabular}

Table 5

Ratios of Fe Lines

\begin{tabular}{|c|c|c|c|c|c|c|}
\hline$\overline{\mathrm{CV}}$ & Fe XXIII 11.43/11.74 & Fe XXII 11.92/11.77 & Fe XVII $15.01 / 15.26$ & Fe XVII $17.10 / 16.77$ & Fe XVII $17.10 / 17.05$ & $\overline{F e}$ XVII $3 \mathrm{~s} / 3 \mathrm{~d}$ \\
\hline V603 Aql & $1.69 \pm 1.05$ & $0.56 \pm 0.39$ & $2.21 \pm 1.28$ & $0.60 \pm 0.54$ & $1.86 \pm 1.32$ & $1.83 \pm 1.03$ \\
\hline AE Aqr & $<0.65$ & $<0.47$ & $<4.88$ & $<0.88$ & $0.14 \pm 0.11$ & $<1.47$ \\
\hline TT Ari & $1.28 \pm 1.17$ & $<0.46$ & $8.27 \pm 5.30$ & $1.94 \pm 1.40$ & $6.14 \pm 5.00$ & $1.38 \pm 0.80$ \\
\hline V834 Cen & $1.64 \pm 1.14$ & $6.26 \pm 5.80$ & $3.63 \pm 3.05$ & $<1.07$ & $<0.68$ & $<2.90$ \\
\hline SS Cyg-Q & $1.76 \pm 0.79$ & $0.32 \pm 0.28$ & $5.20 \pm 3.44$ & $0.34 \pm 0.15$ & $0.32 \pm 0.14$ & $1.85 \pm 0.57$ \\
\hline SS Cyg-O1 & $<0.25$ & $1.14 \pm 0.85$ & $3.85 \pm 1.54$ & $0.46 \pm 0.43$ & $0.51 \pm 0.38$ & $1.17 \pm 0.53$ \\
\hline SS Cyg-O2 & $<0.32$ & $<6.11$ & $1.26 \pm 0.45$ & $0.94 \pm 0.64$ & $0.97 \pm 0.62$ & $2.72 \pm 1.02$ \\
\hline YY Dra & $1.44 \pm 0.94$ & $<2.56$ & $3.74 \pm 1.58$ & $0.30 \pm 0.19$ & $0.36 \pm 0.21$ & $1.17 \pm 0.53$ \\
\hline UG Gem-Q & $1.05 \pm 0.43$ & $2.70 \pm 1.87$ & $10.68 \pm 6.67$ & $0.19 \pm 0.10$ & $0.15 \pm 0.10$ & $1.01 \pm 0.53$ \\
\hline UG Gem-O & $1.10 \pm 0.82$ & $0.45 \pm 0.40$ & $3.50 \pm 3.48$ & $1.23 \pm 1.18$ & $0.62 \pm 0.56$ & $0.94 \pm 0.43$ \\
\hline AM Her & $1.31 \pm 1.22$ & $1.82 \pm 1.43$ & $1.00 \pm 0.97$ & $<0.89$ & $0.88 \pm 0.85$ & $0.51 \pm 0.34$ \\
\hline EX Hya2000 & $1.46 \pm 0.86$ & $1.29 \pm 0.71$ & $2.87 \pm 0.76$ & $0.07 \pm 0.04$ & $0.05 \pm 0.02$ & $1.11 \pm 0.20$ \\
\hline EX Нyа2007 & $1.21 \pm 0.18$ & $1.29 \pm 0.71$ & $3.05 \pm 0.36$ & $0.05 \pm 0.04$ & $0.05 \pm 0.04$ & $1.22 \pm 0.12$ \\
\hline WX Hyi & $2.23 \pm 1.46$ & $1.65 \pm 1.33$ & $3.64 \pm 2.15$ & $0.87 \pm 0.59$ & $1.00 \pm 0.77$ & $1.84 \pm 1.01$ \\
\hline GK Per-1 & $<0.79$ & $<0.28$ & $<1.73$ & $1.67 \pm 0.91$ & $3.87 \pm 2.60$ & $<0.43$ \\
\hline GK Per-2 & $<1.52$ & $<0.47$ & $<0.98$ & $<2.26$ & $1.84 \pm 1.74$ & $0.32 \pm 0.26$ \\
\hline GK Per-Av & $0.88 \pm 0.80$ & $<1.47$ & $<3.87$ & $<3.10$ & $1.24 \pm 1.16$ & $0.18 \pm 0.12$ \\
\hline AO Psc & $<1.53$ & $2.83 \pm 2.54$ & $<1.64$ & $<1.50$ & $<0.95$ & $0.79 \pm 0.59$ \\
\hline V1223 Sgr & $1.59 \pm 1.43$ & $0.89 \pm 0.66$ & $<0.81$ & $2.46 \pm 2.12$ & $1.69 \pm 1.42$ & $0.71 \pm 0.48$ \\
\hline SU UMa & $<5.25$ & $<0.19$ & $1.28 \pm 1.21$ & $1.13 \pm 0.69$ & $0.86 \pm 0.61$ & $0.73 \pm 0.43$ \\
\hline
\end{tabular}

ionization temperature range of $\sim 0.6$ to $\sim 1.1 \mathrm{keV}$, based on the error bars, with a mean ionization $T_{\mathrm{e}}$ of $\sim 0.9 \mathrm{keV}$ when comparing the observed point to the solid curve.

Also note that the error bars are very large in many cases or that there are numerous upper limits. We note that for errors to be small relative to the value of the ratio, the errors on the individual quantities comprising the ratio need to be even smaller. As noted in Shipley et al. (2015), many of the CV grating spectra are underexposed. Rather than dropping or hiding ratios with upper limits or large errors, we elected to exhibit all of the data.

\subsection{Temperature Measures}

In general, measures of temperature originate from ratios of line strengths in which the upper levels have signifi- cantly different excitation energies; if the lines appear in the same waveband, the simultaneous measure provides the diagnostic. Particular ratios lead to ionization temperatures (e.g., $\mathrm{H}-/ \mathrm{He}-\mathrm{K} \alpha$ ), and excitation temperatures (e.g., triplet lines). This section examines the $\mathrm{H}-/ \mathrm{He}-\mathrm{K} \alpha$, triplet $G$, and Fe XVII line ratios.

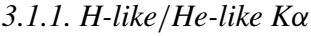

The ionization temperature of the emission regions, as measured by the H-like to He-like K $\alpha$ ratio, is shown in Figure 2(a), for $\mathrm{Mg}$ and $\mathrm{Fe}$, and Figure 2(b) for $\mathrm{O}$ and $\mathrm{S}$. The curves for $\mathrm{Si}$ and $\mathrm{Ne}$ are not included, but they exhibit similar parallel behavior; the $\mathrm{Si}$ curve lies halfway between the $\mathrm{S}$ and $\mathrm{Mg}$ curves; $\mathrm{Ne}$ lies near the halfway point between $\mathrm{Mg}$ and $\mathrm{O}$. The solid curves 


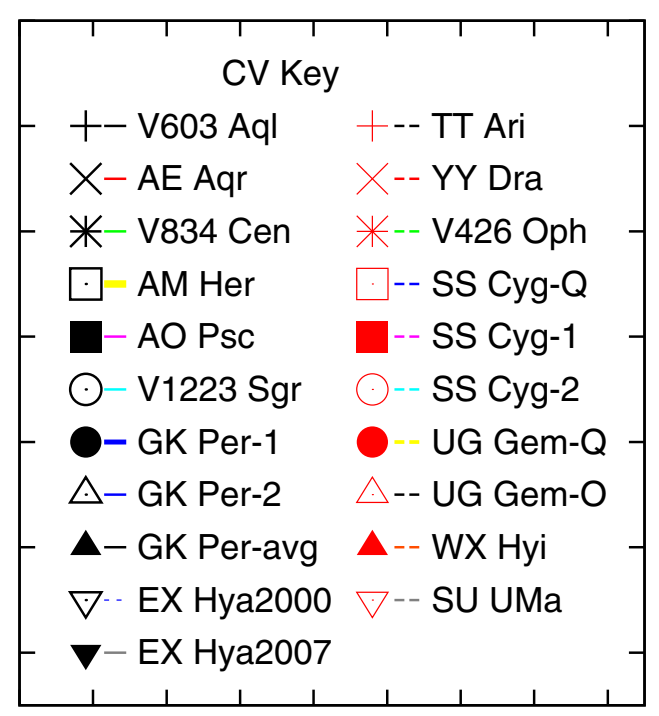

Figure 1. Key to CV plot symbols. These symbols are used in all of the plots. Black points are magnetic CVs; red points are nonmagnetic CVs. (A color version of this figure is available in the online journal.)

slanting from the lower left to the upper right represent the loci of points for collisional ionization equilibrium (CIE); the nearly parallel, dotted curves (for $\mathrm{Mg}, \mathrm{S}$, and $\mathrm{Fe}$ ) represent the neglect of forbidden emission in the calculation. This neglect is equivalent to assuming the high-density limit (e.g., Mewe et al. 1985). Given the size of the typical error bar, in general, we cannot ascertain whether departures from CIE exist on the basis of these plots (but see Section 3.4).

A broad range of ionization temperatures exists, from $\sim 2$ to $30 \mathrm{keV}$ or more, based on the Fe line ratio. The temperature range for nonmagnetic CVs may extend to lower values; however, within the errors, the mean values overlap.

For EX Hya2007, a 0.5 Ms exposure of EX Hya, the errors on the line ratio are sufficiently small to distinguish a temperature range for CIE versus $\mathrm{CIE}$ minus forbidden emission: for $\mathrm{Fe}$ and for strict CIE, the ratio indicates an ionization temperature range of $\sim 9-10 \mathrm{keV}$ while for CIE minus forbidden emission, the range is $\sim 6-8 \mathrm{keV}$. For $\mathrm{Mg}$, the line ratio indicates a temperature of $\sim 1.3-1.5 \mathrm{keV}$ (CIE) versus $\sim 0.85-0.95 \mathrm{keV}$. In principle, $\mathrm{X}$-ray line ratios will deliver measurements of the necessary parameters to increase our understanding of the physics.

In general, the cooling flow models cover a similar temperature range: for example, models match the observed ratios for oxygen for temperatures of $\sim 0.5$ to $\sim 5 \mathrm{keV}$. To match the observed range, a higher upper temperature is required for $\mathrm{S}$ and Fe.

Photoionization models present a different challenge: a large number of parameters may plausibly affect the emission, including $\log \xi$ (ionization parameter), the number density $\left(N_{\mathrm{e}}\right)$, the thermal temperature $(T)$, and the radiation temperature $\left(T_{\mathrm{rad}}\right)$. Consequently, models are degenerate in predicted line emission ratios if a limited set of parameters are varied. For example, based on the oxygen $\mathrm{H}$-/He-like ratio, plausible parameter ranges include a high density $\left(10^{16}\right)$, low temperature $\left(10^{5} \mathrm{~K}\right)$ region as well as a low-density $\left(10^{12}\right)$ region. A full comparison of photoionization models against the observed line ratios to determine the degree of degeneracy lies outside the scope of this paper. The models adopted for this paper are illustrative and are not intended to explore parameter space.

\subsubsection{Figure 3: Fe XVII}

Additional temperature measures are available using the Fe XVII lines. The ratio of Fe XVII 15.01-15.26 $(0.81-0.82 \mathrm{keV})^{9}$ is correlated with electron temperature (Brown et al. 2001; Figure 3). The curve, as described by Brown et al. (2001), originates with ion balance calculations of Arnaud $\&$ Raymond 1992). While the error bars are large in general, the data clearly demonstrate a range of temperatures from $\sim 1$ to 4 MK $(\sim 0.1-0.4 \mathrm{keV})$; the value for the ratio for Capella indicates a similar temperature range. Gillaspy et al. (2011) have refined the measurements and the theoretical curve and demonstrate that oscillations exist in the $G$ ratio at low values. We do not include the oscillatory behavior because the signal-to-noise in the data do not warrant the extra detail at this time.

The entire set of lines of the Fe XVII $3 s / 3 d$ levels ${ }^{10}$ defines a temperature measure (Chen $\&$ Pradhan 2005) where the ratio is defined as

$$
\frac{I(16.77+17.05+17.10 \AA)}{I(15.01+15.26+15.45 \AA)} .
$$

The $15.45 \AA$ emission line is an atomically weak line with an expected line strength perhaps $3 \%-4 \%$ and $10 \%$ of the $15.01 \AA$ and $15.26 \AA$ lines, respectively (Doron \& Behar 2002). At the observed signal-to-noise ratio for all of the $\mathrm{CVs}$, the contribution of the $15.45 \AA$ line may be ignored because, in general, we do not detect the $15.45 \AA$ line and it has a small effect on the resulting ratio (Figure 3 ). The ratios yield a minimum temperature of $\log$ $T_{\mathrm{e}} \sim 6.1$. For GK Per, the ratio implies temperatures larger than $\log T_{\mathrm{e}}=7.2$. For SS Cyg and U Gem, CVs with outburst and quiescence spectra, no difference is apparent in the X-ray line ratios between the states.

The average values of the ratio differ slightly between the two types of CVs (magnetics: 0.84; nonmagnetics: 1.33), but are consistent within the errors. We are uncertain of the weight to place on the separation of the average values, given our small-number statistics. The implication is clearly worthy of confirmation with a set of line measurements with higher signalto-noise from a greater number of CVs. The Fe XVII ratios (Figure 3) also represent one of the only diagnostics presented in this paper in which an observable difference may exist between the magnetic and nonmagnetic CVs (but see also Section 3.5).

That several of the values lie below the line for the magnetic CVs was a puzzle until the publication by Bernitt et al. (2012). They deduce unexpectedly low oscillator strengths as the source of differences between observations and predictions for Fe XVII line emission. The predicted values are up to $\sim 50 \%$ above the measured values.

\subsubsection{Triplet G Ratios}

The final temperature measure is the triplet $G$ ratio first described by Gabriel \& Jordan (1969). This measure of the electron temperature originates with the ratio of the resonance $\left(r, 1 s^{2}{ }^{1} S_{0} \rightarrow 1 s 2 p{ }^{1} P_{1}\right)$, intercombination $\left(i, 1 s^{2}{ }^{1} S_{0} \rightarrow 1 s 2 p\right.$ $\left.{ }^{3} P_{2,1}\right)$, and forbidden $\left(f, 1 s^{2}{ }^{1} S_{0} \rightarrow 1 s 2 s^{3} S_{1}\right)$ lines and is defined as $G=(f+i) / r$. Theoretical $G$ ratio curves have been published by a number of authors (Godet et al. 2004; Bautista \& Kallman 2000; Porquet \& Dubau 2000).

\footnotetext{
9 In the literature, this ratio is referred to as "3C"/"3D."

10 The energy range for these lines is $\sim 0.72-0.82 \mathrm{keV}$.
} 

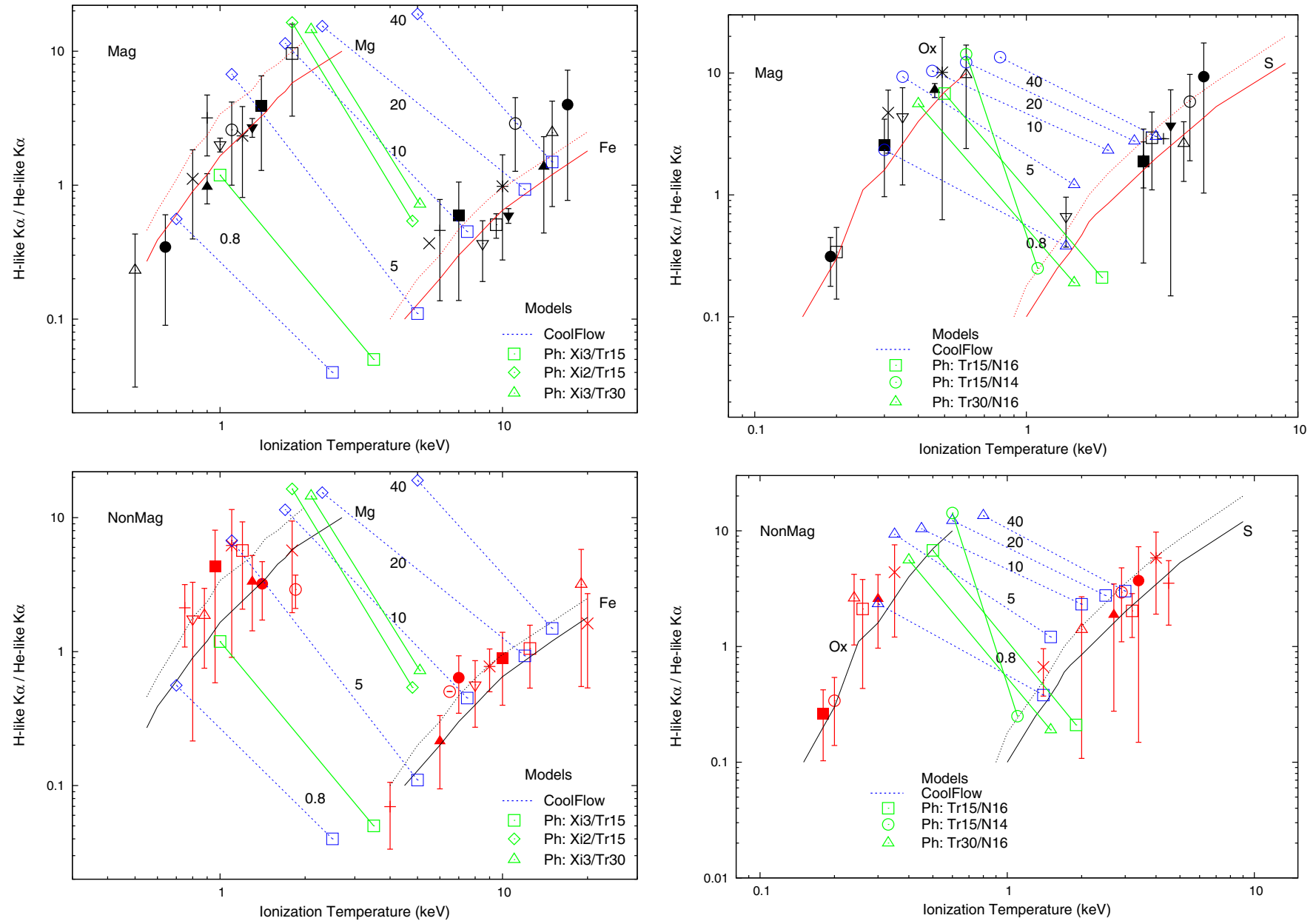

(a)

(b)

Figure 2. $\mathrm{H}$-like $\mathrm{K} \alpha$ to He-like $\mathrm{K} \alpha$ ratio for (a) $\mathrm{Mg}$ and $\mathrm{Fe}$; and (b) $\mathrm{O}$ and $\mathrm{S}$ for (top) magnetic and (bottom) nonmagnetic CVs. Lines for Si and Ne are parallel but have been omitted for clarity. In this and all subsequent plots, the data points are plotted by emission line identity, but are arbitrarily placed around the line solely to distribute the points for visibility and aid in the identification of a particular CV. Solid red curves, trending from lower left to upper right, are the loci corresponding to collisional ionization equilibrium; the nearly parallel, dotted red curves are the loci of models that neglect forbidden emission, essentially corresponding to the high-density limit. Blue symbols represent line ratios obtained from "cooling flow" models (e.g., Mukai et al. 2003), with the dashed lines connecting ratios obtained from the same model. The lines are labeled (in black) with the value of the $T_{\max }$ parameter of the cooling flow model; the labels range from 0.8 to $40 \mathrm{keV}$. Green points represent photoionization models as labeled in the key. Photoionization models have at least four to five significant parameters and the plots capture a measure of the variations. For the $\mathrm{Fe} / \mathrm{Mg}$ plot, the photoionization parameters are the $\log$ ionization $(\log \xi)$ and the radiation temperature $\left(T_{\text {rad }}\right)$. For the $\mathrm{S} / \mathrm{O}$ plot, the photoionization parameters are the radiation temperature and the log number density $(N)$. Note that plausible "cooling flow" and photoionization models generate similar line ratios. In this and all subsequent plots, and because of the underexposure (see Shipley et al. 2015) present in almost all of the grating spectra, inferences about the measurements will refer to the data points with the smallest errors only. (b) $\mathrm{H}$-like $\mathrm{K} \alpha$ to $\mathrm{He}$-like $\mathrm{K} \alpha$ ratio for $\mathrm{O}$ and $\mathrm{S}$.

(A color version of this figure is available in the online journal.)

Figure 4 displays the values of the ratio. The lines on the plots show the loci of models for coronal and photoionization conditions (solid and dashed, respectively). ${ }^{11}$

Specific ratios for individual CVs provide restrictions. The Fe XXV ratios for U Gem, one epoch of WX Hyi, and AO Psc are sufficiently low that they are consistent with either coronal

11 Technically, the $G$ and $R$ ratios (next sub-section) should include the effects of satellite lines (e.g., Porquet et al. 2010), so that the definition of the $G$ ratio becomes

$$
G=\frac{\left(z+\text { satellite } \_z\right)+\left(x+y+\text { satellite } \_x y\right)}{w+\text { satellite } \_w}
$$

where the more familiar $f, i, r=z, x+y, w$. Similarly, for $R$

$$
R=\frac{z+\text { satellite } z z}{(x+y)+\text { satellite } \_x y} .
$$

As noted in Shipley et al. (2015), we do not include satellite line emission because it is generally weak in the CVs in which it could be measured. or photoionized conditions at temperatures $\gtrsim 10^{7} \mathrm{~K}$. In contrast, EX Hya is consistent either with very low temperature coronal conditions, $T<10^{6} \mathrm{~K}$, or with photoionized conditions at $T \sim 10^{7.2} \mathrm{~K}$. Hoogerwerf et al. (2005) conclude that the WD in EX Hya is insufficiently luminous to generate photoionization conditions, leading to a preference for the value corresponding to low-T coronal conditions.

For Si XIII, all of the lines are consistent with $T \gtrsim 10^{6} \mathrm{~K}$; those points with the smallest errors are consistent with $T \gtrsim 10^{6.8} \mathrm{~K}$, a temperature at which the coronal and photoionized conditions are not separable. The S Xv ratios generally show large errors; those ratios with the smallest errors indicate coronal conditions in the range $5.6<\log T<6.4$ or photoionized conditions a $T \sim 10^{7} \mathrm{~K}$.

The $G$ ratio results may be summarized by noting that the values point to temperatures, regardless of mechanism, in the $5.6<\log T(\mathrm{~K})<7.0$ range. In general, the $G$ ratio does not separate magnetic from nonmagnetic CVs. 


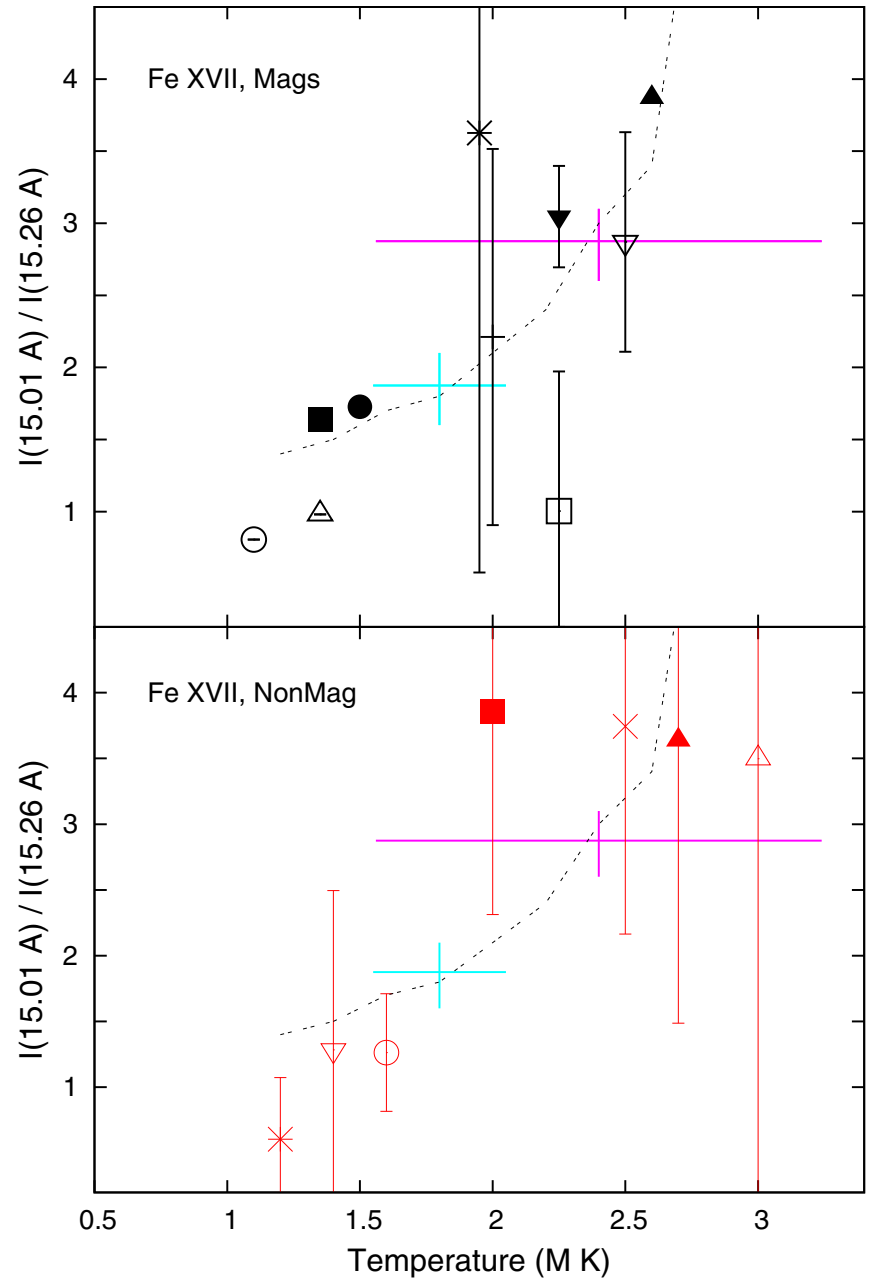

(a)

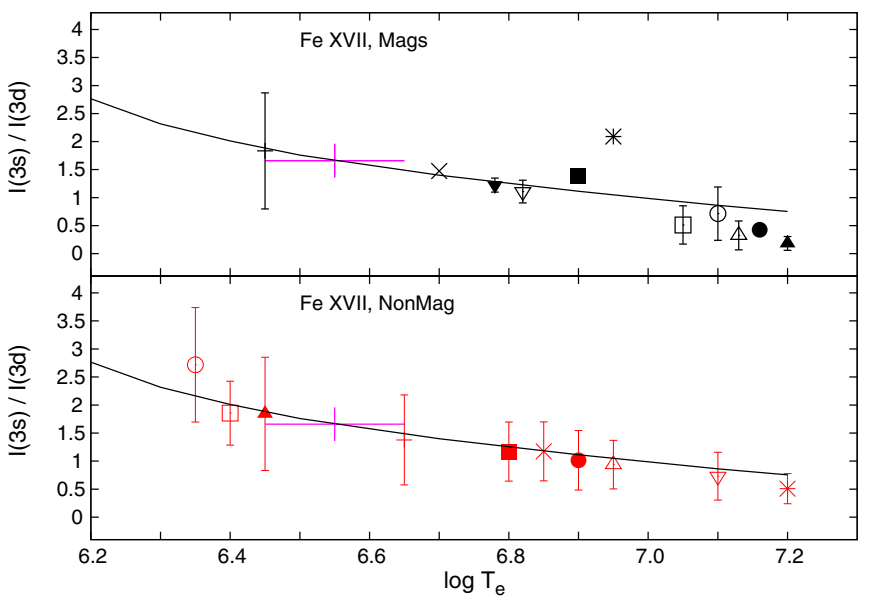

(b)

Figure 3. (a) Fe XVII 15.01/15.26 Å line flux ratio vs. temperature for the available CVs. Included on the plot is the value for GK Per (XMM-Newton) from (Vrielmann et al. 2005; light blue), and Capella from (Behar et al. 2001; pink). The data points are distributed arbitrarily along the horizontal axis. The curve represents an ion balance calculation of Arnaud \& Raymond (1992). Note that the upper plot for the magnetic CVs has half the scale of the lower plot. (b) Fe XVII 3s/3d line ratio: $I(16.77+17.05+17.10 \AA) / I(15.01+15.26+15.45 \AA)$ vs. temperature using the theoretical curve from Chen \& Pradhan (2005). The Capella point (pink) is from Behar et al. (2001). Temperature ranges for a given CV may be obtained by mapping the measures to the curve: e.g., for SS-Q (red open square), the range is $\sim 6.5 \pm 0.3$.

(A color version of this figure is available in the online journal.)

\subsection{Satellite Lines and Temperature}

Gabriel \& Phillips (1979) noted that the presence of satellite lines could be used as a measure of electron temperature. Our fits only reveal the " $j$ " line (line notation as in Gabriel 1972) for two lines (Mg and $\mathrm{Si}$ ) of three CVs (AM Her, SS Cyg-Q, and EX Hya2007). Figure 5 shows the results using the fits from Shipley et al. (2015). The curve asymptotically approaches a $j / w$ ratio of $\approx 0.1$. Consequently, the EX Hya2007 points fall well below the temperature curve-that they do so likely indicates a problem in setting the continuum level. For $\mathrm{Mg}$, the $j$ line represents $\sim 1 \%$ of the flux of the resonance line; for $\mathrm{Si}$, the ratio is $\sim 2 \%$. A small change in setting the local continuum level can therefore have a large impact on the fitted flux in the $j$ line. This problem does not eliminate the use of the satellite lines as temperature indicators - the method only requires data with a higher signal-to-noise ratio, particularly in the continuum.

\subsection{Number Density Measure}

As with temperature, density measures generally arise from two lines with nearly identical excitation energies. Indicators of number density have been investigated previously, most notably by Mauche et al. $(2005,2001)$ or Liedahl et al. (1992) for the $17.10 / 17.05 \AA$ and triplet $R$ ratios. Those authors discussed the effects of UV contamination on the interpretation of the line ratios. We first review our observed line ratios, then discuss the effects of possible contamination.

\subsubsection{Fe XVII 17.10/17.05; 17.10/16.77}

Figures 6(a) and (b) show the ratios of the line strengths of Fe XVII $17.10 \AA$ to $17.05 \AA(0.72 / 0.73 \mathrm{keV})$ and $17.10 \AA$ to $16.77 \AA(0.72 / 0.74 \mathrm{keV})$. The first ratio was described by Mauche et al. (2001) for EX Hya while the second was presented in Liedahl et al. (1992). Within the errors, the $17.10 / 17.05$ values are all consistent with a number density $>10^{14} \mathrm{~cm}^{-3}$; several of the best-measured magnetics indicate a number density $\sim 10^{15} \mathrm{~cm}^{-3}$, assuming no dependence upon a photoexcitation radiation field of several $10 \mathrm{kK}$ as discussed by Mauche et al. (2001, 2005) or Liedahl et al. (1992).

For the $17.10 / 16.77$ line ratio, the data indicate a minimum number density $\gtrsim 10^{14} \mathrm{~cm}^{-3}$; the points with the smallest errors imply a number density $\gtrsim 10^{14.7} \mathrm{~cm}^{-3}$, a factor of a few above the value measured for Capella. With the current exposures, 

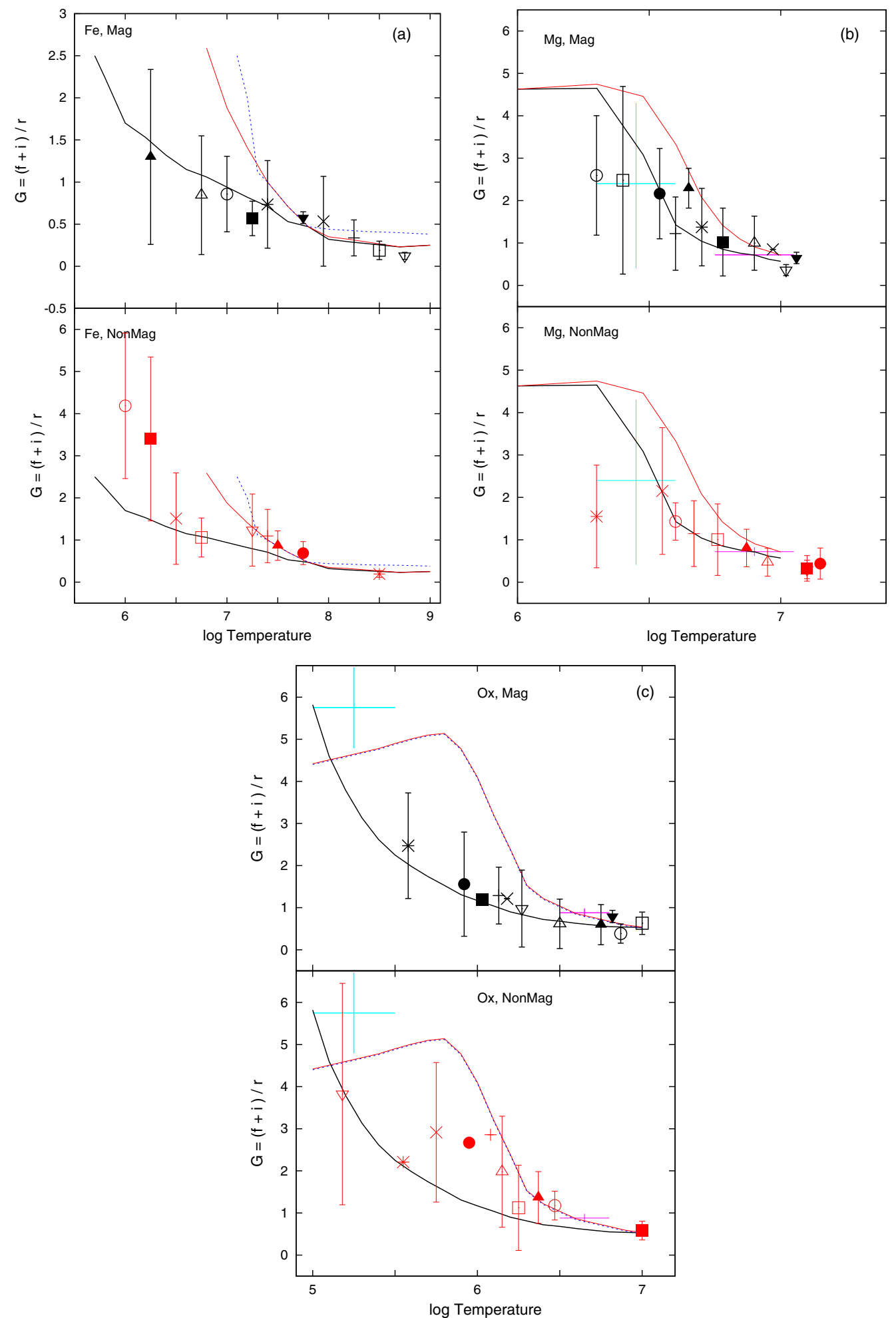

Figure 4. $G=(f+i) / r$ ratios for (top) magnetic CVs and (bottom) nonmagnetic CVs for (a) Fe XXV; (b) Mg XI; and (c) O vII The lines define the loci of models for coronal conditions (solid black), coronal conditions including satellite lines (solid red), and photoionized conditions (dashed blue). The three model curves are not present on all plots: for example, for O, the coronal and photoionized curves are nearly identical. The curves for Fe, Si, S, and O originate from Bautista \& Kallman (2000); the curve for $\mathrm{Mg}$ is in Porquet \& Dubau (2000). The data points are distributed arbitrarily on the horizontal axis for visibility. The pink point in all except Fe represents Capella (Canizares et al. 2000); the light blue point represents the XMM-Newton observation of GK Per (Vrielmann et al. 2005). For Fe, the measurements are generally consistent with high temperatures, hence the merged portion of the curves; only SS Cyg in outburst (red filled square; red open octagon) may distinguish between coronal and photoionization conditions. (b) $G$ ratio for $\mathrm{Mg}$ (see (a) for details). For $\mathrm{Mg}$, the separation between coronal and coronal with satellite emission is too small to separate given the measurement errors. The measured ratios for CVs with small error bars indicate high temperatures, $\log T>6.5$. (c) $G$ ratio for $\mathrm{O}$ (see (a) for details). The photoionization curve has been offset downward by -0.02 for clarity. For O, a narrow range in $\log T(\sim 5.4-6)$ exists in which the $G$ ratio could separate photoionization and coronal conditions. For the magnetic CVs, several (V603 Aql, AE Aqr, V834 Cen, EX Hya2000, GK Per-1) indicate the existence of either coronal conditions at $\log T \sim 5.5$ or photoionization conditions at $\log T \sim 6.3$. For others (AM Her, EX Hya2007, GK Per-2, V1223 Sgr), $\log T \sim 6.5-7$ regardless of the conditions. For the nonmagnetics, only SS Cyg-2 leads to $\log T \sim 5.7$ (coronal) or $\log T \sim 6.3$ (photoionization). For the others, the errors are sufficiently large enough that the ranges overlap.

(A color version of this figure is available in the online journal.) 


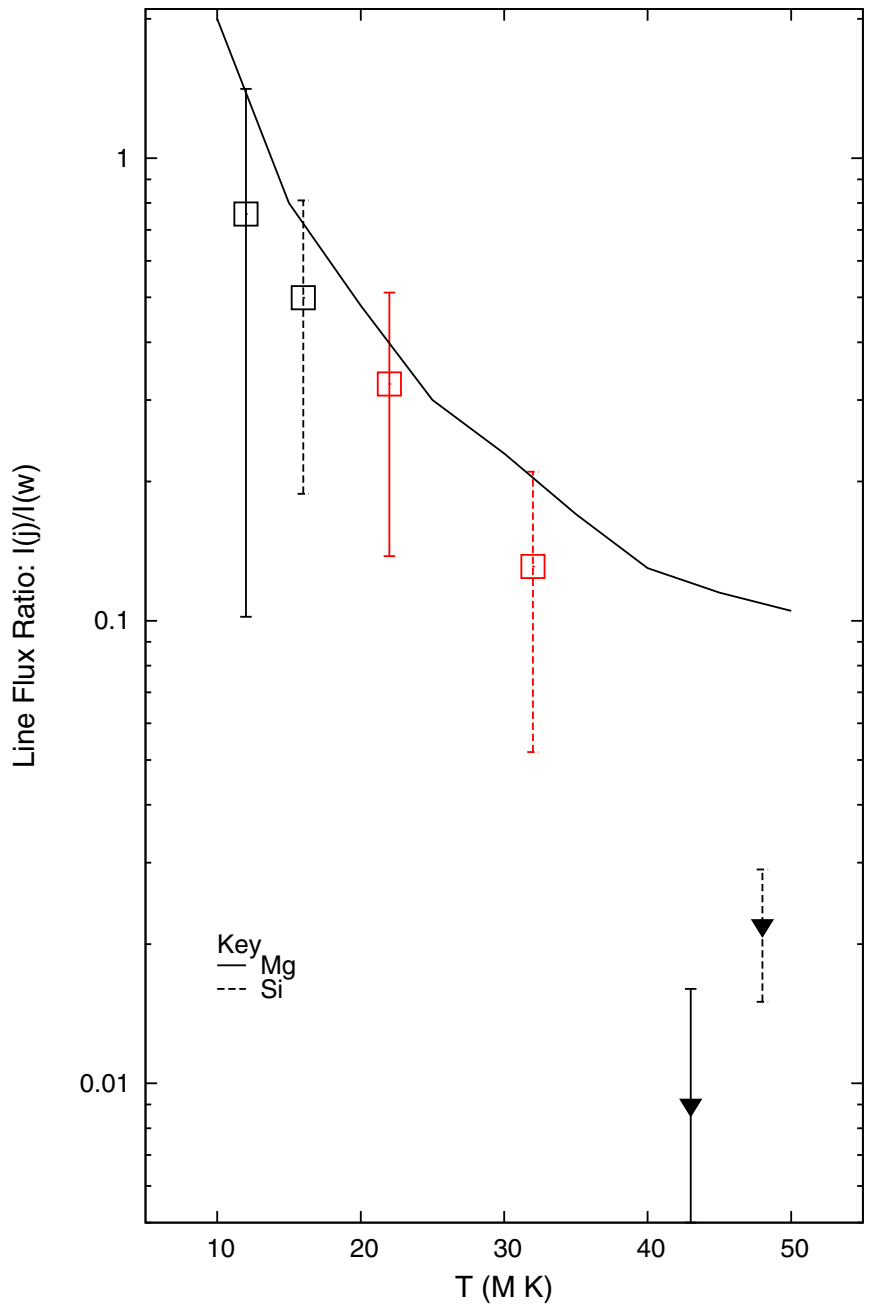

Figure 5. Ratio of line fluxes for the satellite line $j$ and the resonance line vs. temperature (Gabriel \& Phillips 1979). See Section 3.2 for a discussion.

(A color version of this figure is available in the online journal.)

there are insufficient counts to investigate the dependence of the line ratio on changes in electron temperature as described in Liedahl et al. (1992). This ratio could be contaminated by a UV radiation field near a wavelength of $\sim 870 \AA$.

\subsubsection{Triplet $R$ Ratios}

The $R$ ratio, where $R=f / i$, is a density indicator (e.g., Porquet et al. 2010). In the high-density limit, the intercombination line is expected to be stronger than the forbidden line because an electron that is excited to the upper level of the forbidden line $\left({ }^{3} S_{1}\right)$ may be additionally impact-excited to the upper level of the intercombination line. The subsequent decay enhances the intercombination line (see Porquet et al. 2010 for additional details).

Itoh et al. (2006) reported a number density of $\approx 10^{11} \mathrm{~cm}^{-3}$ for $\mathrm{AE} \mathrm{Aqr}$ based on the $\mathrm{He}$-like triplet ratios of $\mathrm{N}, \mathrm{O}$, and $\mathrm{Ne}$. The Ne triplet corresponded to an upper limit on the number density of $10^{13} \mathrm{~cm}^{-3}$; the ratios for $\mathrm{N}$ and $\mathrm{O}$ were consistent with a number density of $10^{10.5-11.5}$. Their values are essentially identical to our inferences for $N_{\mathrm{e}}$ if we restrict our attention solely to AE Aqr.

We obtain a higher value for $N_{\mathrm{e}}$ for the ensemble of CVs. Figure 7 plots the triplet $R$ ratio. Focusing on the most restrictive upper limits, or the data with the smallest errors, and taking the measures as plotted, we note that the $\mathrm{R}$ value for $\mathrm{Fe}$ implies $N_{\mathrm{e}} \gtrsim 10^{17-18} \mathrm{~cm}^{-3}$; for $\mathrm{S}$ and $\mathrm{Si}, \gtrsim 10^{14-15} \mathrm{~cm}^{-3}$; for $\mathrm{Mg}$, $\sim 10^{13-14} \mathrm{~cm}^{-3}$; and for $\mathrm{O}, \sim 10^{12-13} \mathrm{~cm}^{-3}$. This would be an extraordinary range if correct.

However, the fidelity of $R$ as a density indicator may be seriously compromised by a strong UV continuum source as is expected to exist in a typical CV (e.g., Gabriel \& Jordan 1969; Blumenthal et al. 1972; Pradhan \& Shull 1981; Porquet et al. 2001; Mauche et al. 2003a among others). The contamination increases the $R$ values toward the high-density regime as shown previously (e.g., Mauche 2003a for EX Hya; Kahn et al. (2001) or Cassinelli et al. (2001) for the O supergiant $\zeta$ Pup). Simulations with a strong UV contribution "depress" the $R$ curves and decrease the interpretative power of the $R$ ratio.

There is another possibility: Blumenthal et al. (1972), and others subsequently, show that the $R$ ratio exhibits a radial dependence (e.g., Waldron \& Cassinelli 2000; Leutenegger et al. 2006):

$$
R(r)=\frac{R_{\mathrm{obs}}}{1+n_{e}(r) / N_{C}+W(r) \phi / \phi_{C}},
$$

where

$$
W(r)=\frac{\phi}{\phi_{C}}\left[\frac{R_{\mathrm{obs}}}{R(r)}-1\right]
$$

and $\phi=\frac{c^{3}}{8 \pi h v^{3}} U_{v}$ is the photoexcitation rate. This calculation leads to curves that are "depressed" in $R$ as a function of distance from the $U V$ source. This approach has been used in the analysis of, for example, winds of O stars (Waldron \& Cassinelli 2000; Leutenegger et al. 2006). We repeat this analysis here to investigate whether the CV emission is consistent with the WD surface.

Figures 8(a)-(d) show the results for the above calculation for $\mathrm{Si}, \mathrm{Mg}, \mathrm{Ne}$, and $\mathrm{O}$. The curves are very similar to those presented in Waldron \& Cassinelli (2000) or Raasen (2005). The $f / i$ ratios for $\mathrm{Ne}$ and $\mathrm{O}$ indicate that the origin of the emission could lie up to $\sim 10 R_{\mathrm{WD}}$ distant for at least one or two CVs. For Mg, the emission could originate up to $\sim 5 R_{\mathrm{WD}}$. Particularly for the nonmagnetic systems, the X-ray emission region could very well be located in a wind-like flow off the disk, as originally suggested for SW Sex (Honeycutt et al. 1986). Such a geometry implies that a very high signal-to-noise observation should reveal eclipse behavior in the emission lines. Observations of high fidelity could test whether some of the line emission occurs well away from the WD surface.

\subsubsection{Fe XXII $11.92 / 11.77$}

Figure 9 shows the Fe XXII $11.92 \AA / 11.77 \AA(1.04 / 1.05 \mathrm{keV})$ ratio versus number density and compares the theoretical lines of Mauche et al. (2003a) and Liang \& Zhao (2008). Note that the curves in Liang \& Zhao (2008) describe the ratio Fe XXII $(11.92 \AA+11.94 \AA) / 11.77 \AA$. For the $\mathrm{CVs}$, none reveal $11.94 \AA$ emission, thus the two curves may be compared directly.

The points with the smallest errors imply $\log N_{\mathrm{e}} \lesssim 14$ (magnetics) and $\lesssim 13.5$ (nonmagnetics). Note that the values derived from the Fe XXII ratio lie at the low end of the range inferred from the Fe XVII 17.10/17.05 and the triplet $R$ ratio (Section 3.3.2). Given the likely contamination by the UV radiation field for the triplet ratio, it may be possible to use other lines, e.g., the Fe XXII ratio, to infer a density as noted by Mauche et al. (2003a). It may also be possible to iterate a model between the triplet $R$ 


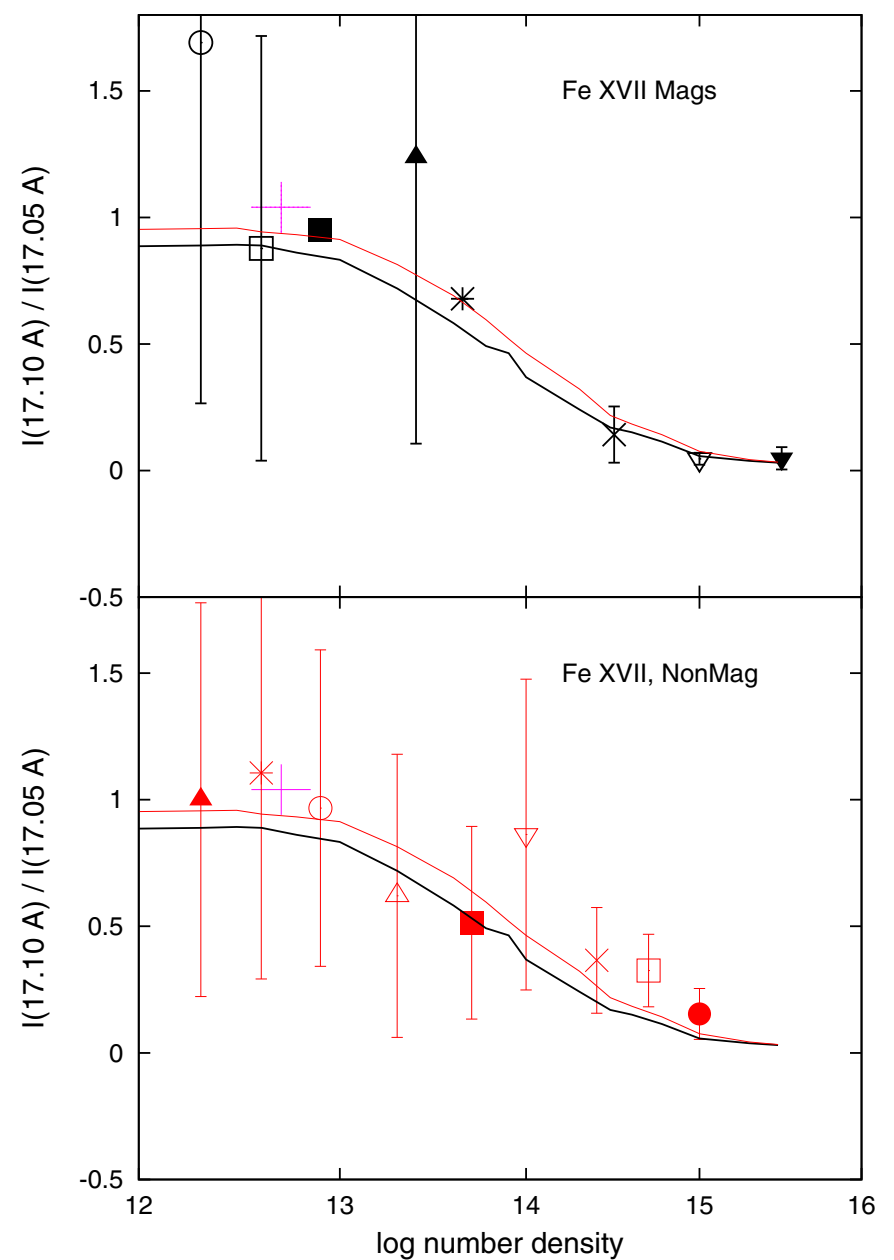

(a)

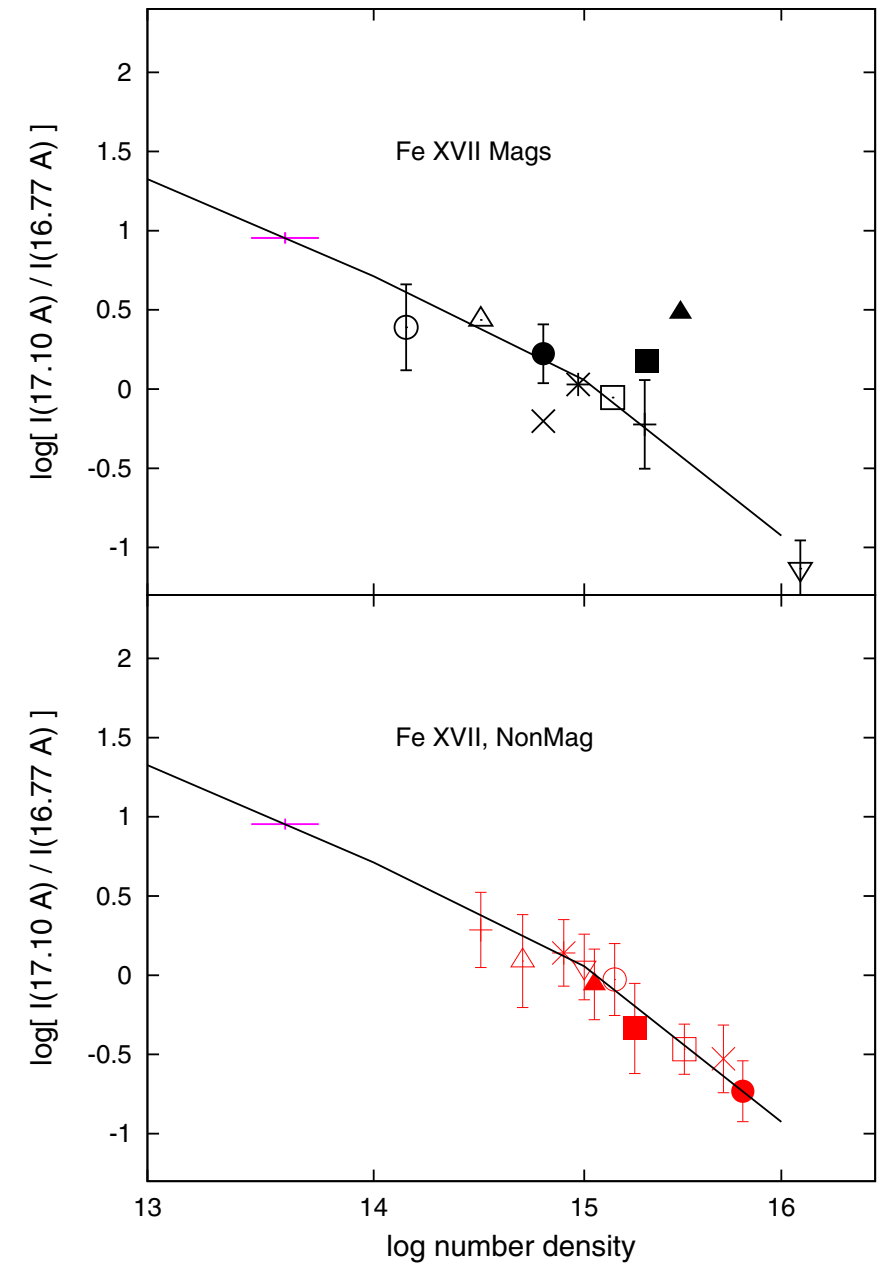

(b)

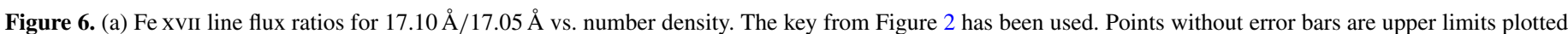

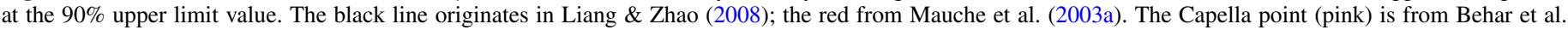

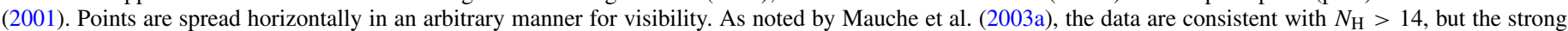

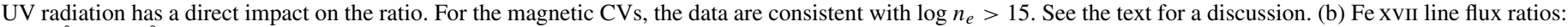

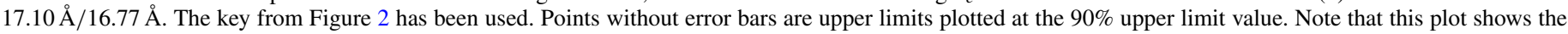

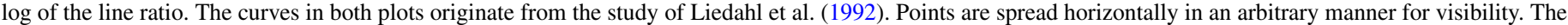
data again indicate high densities $\left(N_{\mathrm{H}}>14\right)$.

(A color version of this figure is available in the online journal.)

and ratios such as Fe XXII to infer a real measure of the number density, in spite of the UV contamination. A small pilot study of that approach is in progress.

As a side note, the transition leading to the Fe XXII $11.92 \AA$ line is not detected in the Sun (Landi \& Phillips 2005), Her X-1 (Jimenez-Garate et al. 2005), or Capella (Canizares et al. 2000).

\subsection{CIE versus NIE}

Liedahl (1999) specified the conditions under which one expects CIE versus nonionization equilibrium (NIE): electron temperatures inferred from the $G$ ratio of the triplet lines should equal ionization temperatures inferred from the $\mathrm{H}$-like $\mathrm{K} \alpha / \mathrm{He}$ like $\mathrm{K} \alpha$ ratio for CIE. Figure 10 shows the combined plot of the triplet $G$ and $\mathrm{H}-\mathrm{K} \alpha / \mathrm{He}-\mathrm{K} \alpha$ curves for $\mathrm{Fe}, \mathrm{S}, \mathrm{Mg}$, and $\mathrm{O}$ along with the observations of those CVs with the smallest errors. In these plots, the black (magnetic) or red (nonmagnetic) lines represent the $G$ ratios while the green lines represent the H-like/ He-like $\mathrm{K} \alpha$ behavior. A similar color assignment is used for the observations. To avoid clutter, only those CVs with smaller error values are plotted-which leads to a nonuniform set of data points for the two curves, but we deem it acceptable to illustrate the temperature ranges.

For $\mathrm{Fe}$, the measured line ratios are broadly consistent with each other, implying that $\mathrm{Fe}$ is in ionization equilibrium. For example, consider EX Hya2007 (inverted filled triangle): the $\mathrm{G}$ value implies a $\log$ temperature $(\mathrm{keV})$ range of $~ 7.5-7.8$; the $\mathrm{H} / \mathrm{He}$ ratio leads to a $\log T$ range of $\sim 7.9-8$, suggesting approximate CIE conditions. The data for $\mathrm{Mg}$ suggest a Gdetermined $\log T$ of $\sim 7$ but, an $\mathrm{H} / \mathrm{He}$-determined $\log T$ of $>7.5$, implying at least partial NIE conditions exist.

For the nonmagnetics, similar conclusions are inferred. For SS-Q (open box), the Fe G-determined $\log T$ is $\sim 6.1-7.4$, while the $\mathrm{H} / \mathrm{He}$-determined $\log T$ is $\sim 8-8.3$. The steepness of the $\mathrm{Mg}$ $\mathrm{G}$ curves leads to a narrow $\log T$ range, $\sim 6.5-6.7$; the $\mathrm{H} / \mathrm{He}$ range is equally narrow, but higher: $\log T \sim 7.4-7.6$.

For oxygen, however, determining whether the coronal or photoionization conditions prevail leads to substantially differing inferences. The $\log T$ range from the $\mathrm{H} / \mathrm{He}$ ratio is $\sim 6.5-6.7$. The inferred range from the $G$ ratio under coronal conditions is $\log T \sim 5.7-6$ again suggesting NIE conditions, but the gas is 

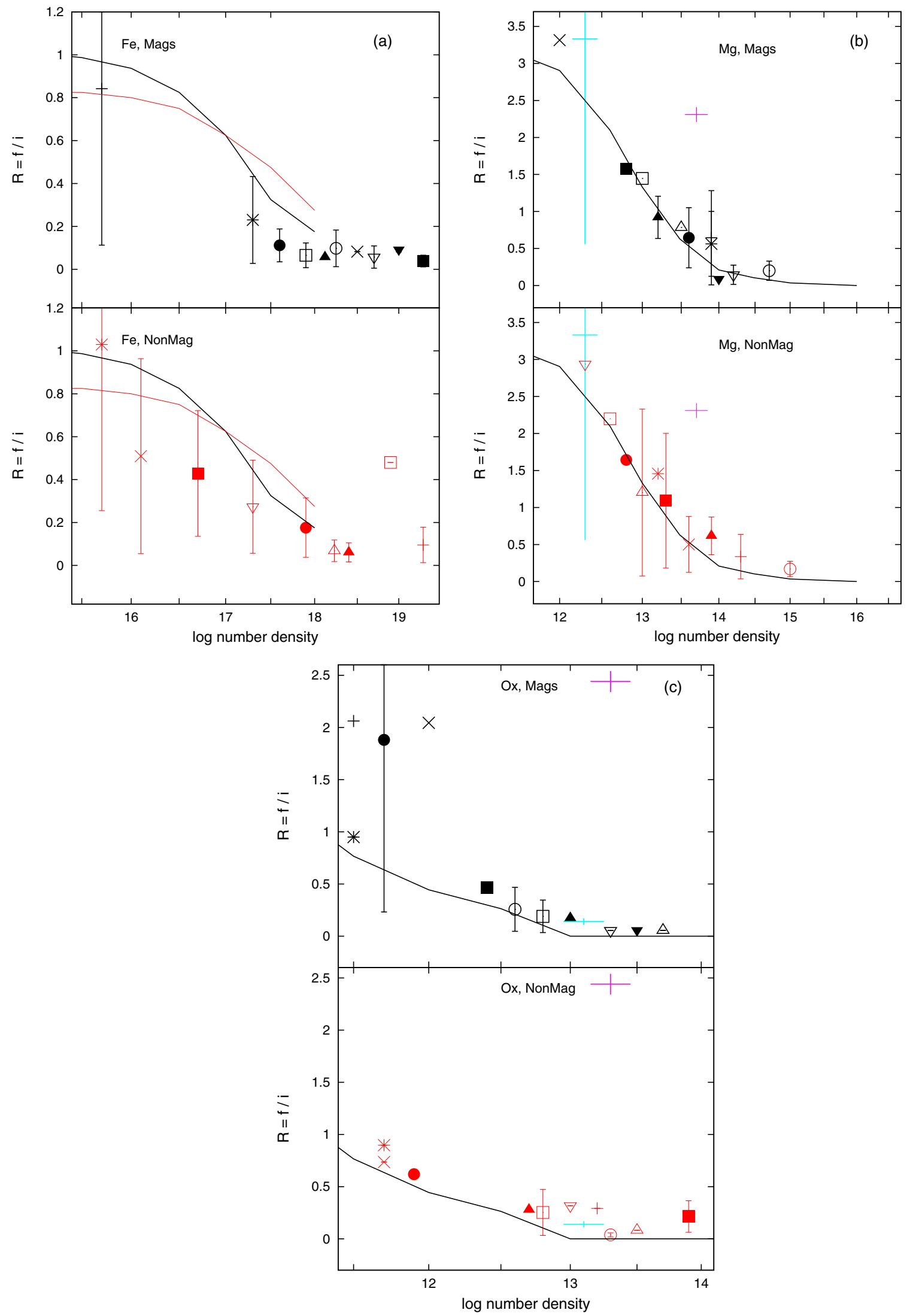

Figure 7. $R=f / i$ ratios for (top) magnetic CVs and (bottom) nonmagnetic CVs for (a) Fe XXV; (b) Mg XI; and (c) O vII. Curves originate from the sources listed in Figure 4. The pink cross in all except the Fe plot represents the position of Capella (Canizares et al. 2000); the light blue cross in the Mg, Ne, and O plots represents the $X M M$-Newton observation of GK Per (Vrielmann et al. 2005). (b) $R$ ratio for Mg. The pink cross in this plot represents the position of Capella (Canizares et al. 2000). The light blue cross in the Mg, Ne, and O plots represents the XMM-Newton observation of GK Per (Vrielmann et al. 2005) (see (a) for details). (c) $R$ ratio for oxygen. The pink cross in this plot represents the position of Capella (Canizares et al. 2000). The light blue cross in the Mg, Ne, and O plots represents the XMM-Newton observation of GK Per (Vrielmann et al. 2005; see (a) for details).

(A color version of this figure is available in the online journal.) 

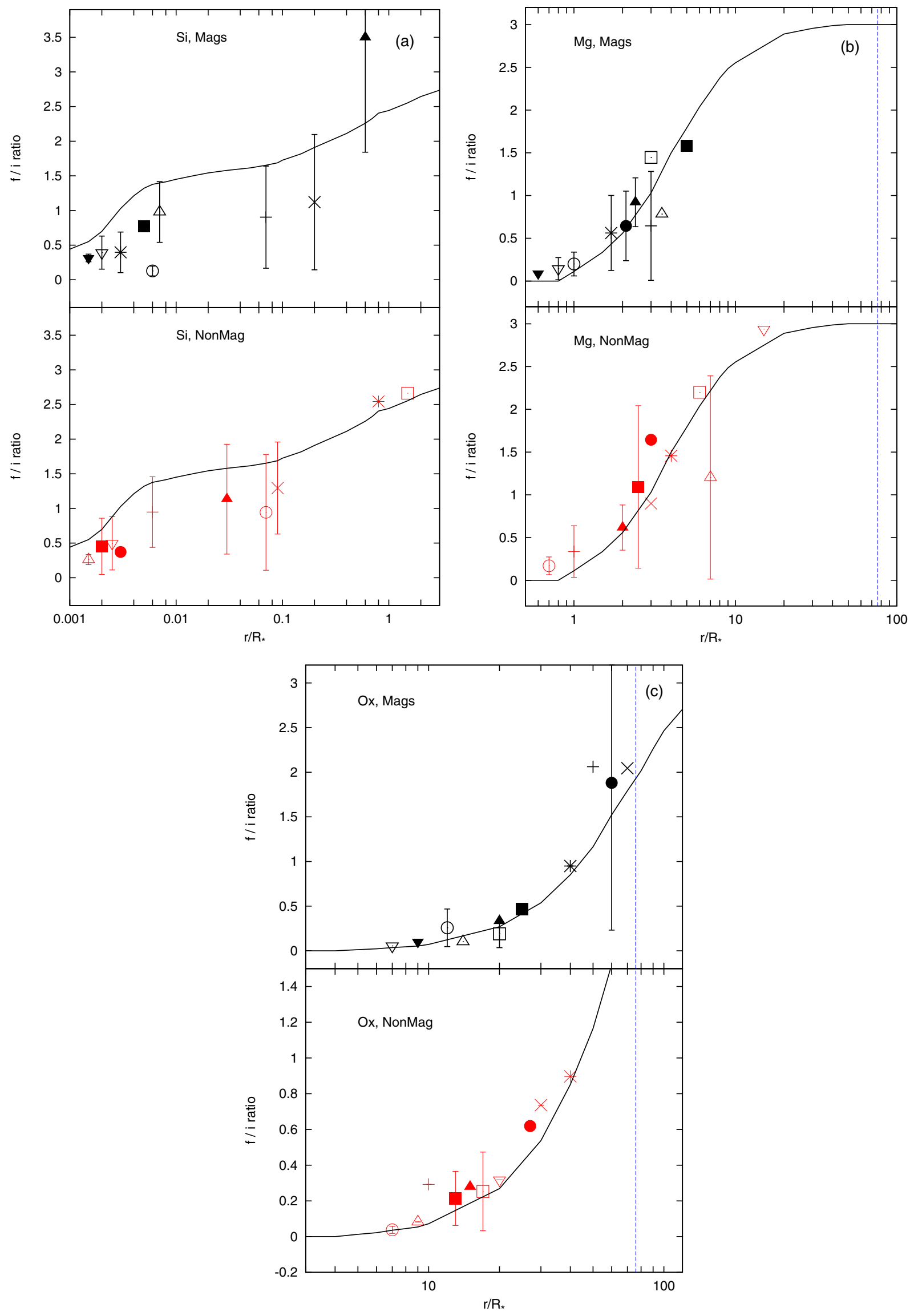

Figure 8. $R$ ratio diluted by distance from the UV source for (a) $\mathrm{Si}$; (b) $\mathrm{Mg}$; and (c) oxygen. (b) $R$ ratio diluted by distance from the UV source- $\mathrm{Mg}$. The vertical blue line in this and the remaining plots represents the size of the orbit for a $\mathrm{CV}$ with a period of $1.5 \mathrm{hr}$. (c) $R$ ratio diluted by distance from the $\mathrm{UV}$ source - oxygen. (A color version of this figure is available in the online journal.) 


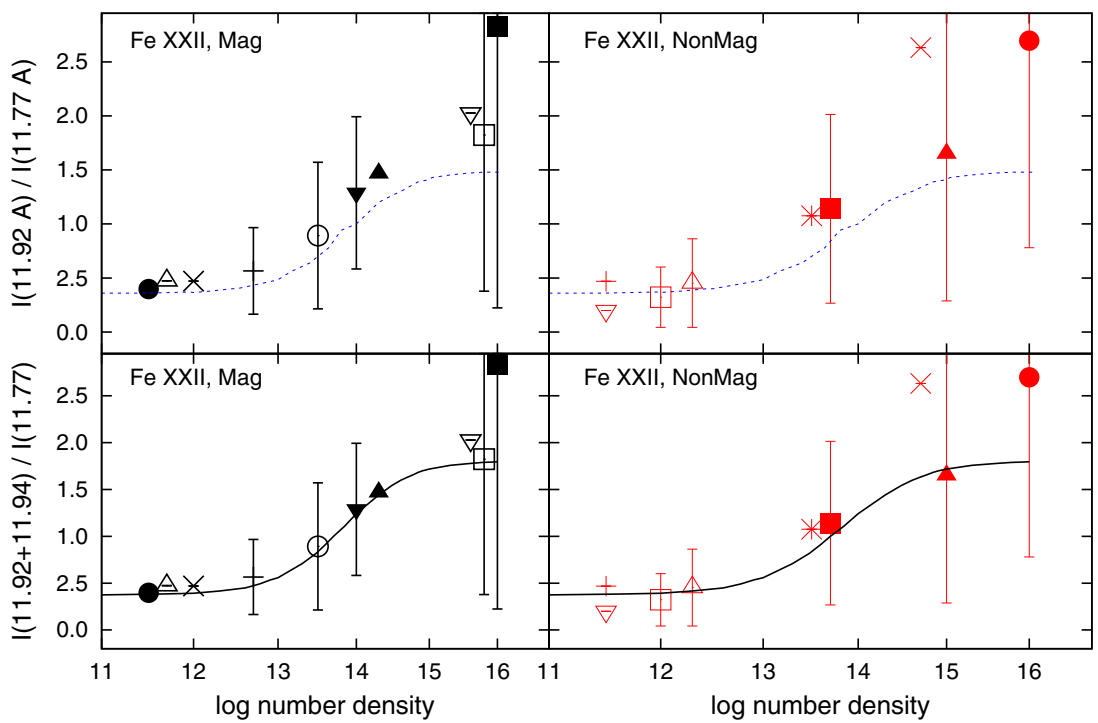

Figure 9. Fe XxII line flux ratios for 11.92/11.77 Å. The key from Figure 2 has been used. Black curve from Liang \& Zhao (2008); blue curve from Mauche et al. (2003a). Points are distributed arbitrarily in the horizontal axis. A point for Capella is not included because the Capella spectrum reveals zero $11.92 \AA ̊$ emission (Behar et al. 2001).

(A color version of this figure is available in the online journal.)

predominantly photo-ionized, then the inferred range is higher, with $\log T \sim 6.3-6.6$, implying CIE conditions.

\subsection{Differences between Magnetic and Nonmagnetic CVs?}

Our sample includes several CV sub-classes, but an insufficient number to divide the data. As noted in Shipley et al. (2015), we can separate the data into two sub-groups, the magnetic and nonmagnetic CVs.

To test for magnetic versus nonmagnetic class-dependent behavior, we folded the line ratio values through a two-sample survival analysis. We chose three cases that appeared to offer the best chance of detecting a difference: the Fe XVII 17.10/16.77 A, the $\operatorname{Mg}$ XI $G$, and the $\mathrm{S}$ XV $R$ ratios. Each of these offered at least a marginal impression of a magnetic versus nonmagnetic separation. None of the three cases indicated a high probability of a difference: the mean values of the probabilities were 0.6 , 0.3 , and 0.3 , respectively - all consistent with no detectable separations. Based on the line ratios presented in this paper, a difference attributed to magnetic or nonmagnetic accretion is not discernible.

\section{DISCUSSION}

What have we learned after surveying all of these results? Our discussion focuses on temperatures, densities, and any separations between magnetic and nonmagnetic CVs.

\subsection{Temperatures}

The argument that a broad range of electron and ionization temperatures exists in CVs based upon CCD-resolution spectra (e.g., Ishida et al. 1994) was an appealing one, but the counter argument, namely, that the spectral resolution prevented an accurate assessment, could always be raised. Were the gas in CVs in isothermal conditions, we would expect identical values for all ions-this is not observed, clearly confirming the Ishida et al. (1994) as well as Mukai et al. (2003) results (Figure 11). The $\mathrm{H} / \mathrm{He}$-determined temperatures range from $\sim 0.2-0.8 \mathrm{keV}$ for oxygen to $\sim 4-20 \mathrm{keV}$ for $\mathrm{Fe}$ (again, using just the observations with the smallest errors). This result also requires future global fits to spectra to be models integrated across a distribution of temperatures.

One firm result of this study is the degree to which the magnetic and nonmagnetic CVs are similar. Consequently, a broad range of temperatures (whether ionization or electron) exists regardless of the details of the accretion process-in other words, the CV subtype does not appear to be a dominant factor (Figure 11). The apparent nondifference essentially confirms the work done fitting WD models to UV spectra of CVs to infer the WD temperature (e.g., Szkody et al. 2007): the disk is not, in general, a significant contributor to the UV radiation field.

\subsection{Densities}

As far as we can ascertain, Hurwitz et al. (1997) was the first, based on EUV data, to estimate a high number density, $\log N_{\mathrm{e}}>15$, for the EUV-emitting regions of CVs. Such a high value fits with theoretical considerations (e.g., Frank et al. 2002) who quote a number density of $10^{16} \mathrm{~cm}^{-3}$.

From X-ray spectra, Mauche et al. (2001) first established a number density estimate of $\sim 10^{14} \mathrm{~cm}^{-3}$ using the Fe XVII 17.10/17.05 A ratio from the observed HETG spectrum of EX Hya. Itoh et al. (2006) argue for a lower number density of $\sim 10^{11-12} \mathrm{~cm}^{-3}$ for AE Aqr because of its special status as a “propeller system" (Venter \& Meintjes 2006).

The ratios presented here provide several different estimates of density. The Fe XXII ratio suggests an upper limit of $\sim 10^{14} \mathrm{~cm}^{-3}$ for the number density, while the triplet $R$ ratio implies that this value is a lower limit.

Both values are almost certainly affected by the WD's strong UV field - this is a well-established theoretical expectation. Consequently, the actual number density must be lower by at least a factor of a few. Unfortunately, any spectral transition is potentially susceptible to the strong UV radiation field, i.e., any transition for which the probability of absorption of a UV photon is comparable to the probability of emission. Hence, any density-sensitive ratio for which the levels are connected is susceptible to UV contamination. 

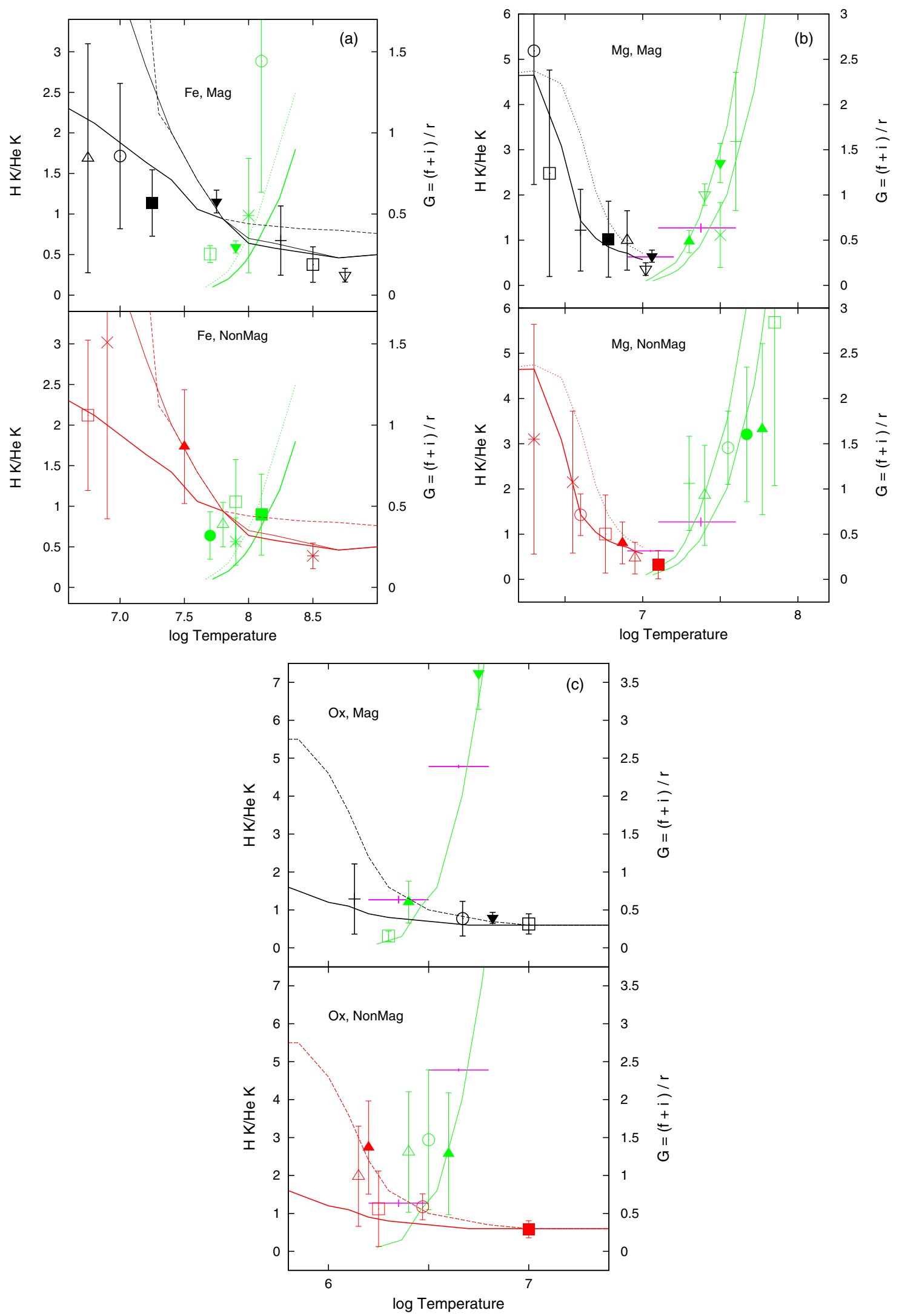

Figure 10. Mash-up of the triplet $G$ and the $\mathrm{H}$-like $\mathrm{K} \alpha / \mathrm{He}$-like $\mathrm{K} \alpha$ ratios. If the inferred temperatures using the two ratios lead to similar temperatures, the plasma exists in CIE conditions. Green values match the right-hand scale but follow the same CV-data point shape prescription of Figure 1. Presented here are (a) Fe; (b) Mg; and (c) $\mathrm{O}$. Here, for Fe, the temperature regimes are similar, indicating CIE conditions. (b) Mash-up of the triplet $G$ and the $\mathrm{H}$-like $\mathrm{K} \alpha / \mathrm{He}-$ like $\mathrm{K} \alpha$ ratio for $\mathrm{Mg}$. The pink points represent Capella. The temperature regimes differ, indicating NIE conditions. (c) Mash-up of the triplet $G$ and the $\mathrm{H}$-like K $\alpha / \mathrm{He}$-like K $\alpha$ ratio for oxygen. The pink points represent Capella. Here, note that the inferred NIE difference is large for the magnetic CVs, but small for the nonmagnetic CVs owing to the generally larger errors on the observed ratios for the nonmagnetic CVs.

(A color version of this figure is available in the online journal.) 


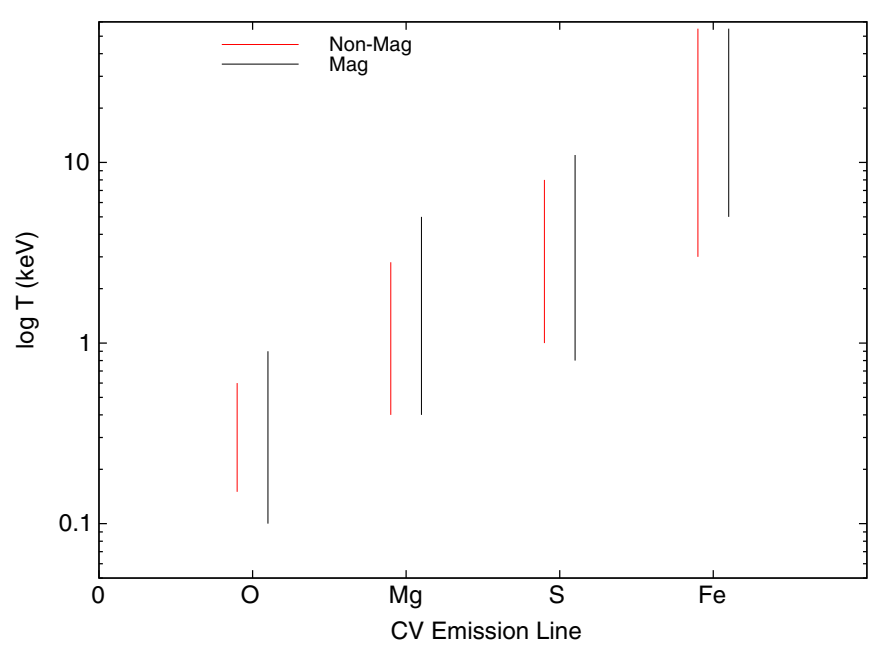

Figure 11. Summary of temperature ranges based on the $\sim 3 \mathrm{CV}$ s of each subclass (magnetic, nonmagnetic) with the smallest error values. For the magnetics, the included CVs are EX Hya (2002 and 2007) and the average GK Per spectrum; and for the nonmagnetics, the included CVs are WX Hyi, SS Cyg (outburst-1), and U Gem (outburst).

(A color version of this figure is available in the online journal.)

As a result, a more concerted approach to estimating the number density may be necessary other than simple line ratios. We have started a small study to examine whether a method exists to extract a sensible estimate of the number density. Basically, we will seek consistency between the degree of contamination and the measured UV spectra of WDs to infer, or place constraints on, a "real" value for $R$.

However, another possibility exists: the ratios are reporting the density accurately, but probe different regions around the $\mathrm{CV}$. This is plausible given the separation between WD and gas implied by the distance-diluted triplet $R$ values. While the Si ratio, and presumably the $S$ and Fe ratios, correspond essentially to the WD surface, $\mathrm{Mg}$ could be slightly elevated above the surface. Neon and oxygen, however, almost certainly lie above the surface, likely by more than a WD radius. Sorting out whether the $R$ ratios are diluted by separation or contaminated by UV radiation will require significant modeling efforts as well as future observations.

\subsection{Photoionization or Not?}

With regard to the debate on photoionization versus "cooling flow" models, the available line spectra do not generally provide sufficient statistics to separate the models. The full range of the $\mathrm{H}-$ and $\mathrm{He}-\mathrm{like}$ ratios for $\mathrm{Fe}$ may be explained by the cooling flow model. For $\mathrm{Mg}$, however, a restricted range is indicated: a high value for the maximum cooling flow temperature leads to essentially zero Mg XI. The Mg XI ion may itself provide a crude measure of temperature-in AM Her, for example, we did not detect $\mathrm{Mg}$ XI.

We note the discussion in Bautista \& Kallman (2000) of photoionization diagnostics, which they label as " $P$ " ratios: transitions from $3{ }^{3} D-2{ }^{3} P$ provide a strong separation between photoionized and thermal line emission. Most of these lines lie longward of the HETG band; only the lines of Fe lie in the HETG band. However, the $3{ }^{3} D-2{ }^{3} P$ lines lie in the $\sim 10-13 \AA$ band where line confusion is significant. Higher spectral resolution in this band will be necessary to sort out the line emission, which can then provide insight to the degree of photoionization. We expect this to be a key focus of observations using X-ray calorimeters on the next generation of X-ray satellites. That would then permit a more complete exploration of the broad range of photoionization parameters - the models are currently degenerate because (1) the ratios have such large errors; and (2) the spectral resolution, while the best available, still leaves critical lines partially blended.

We also did not detect any feature unambiguously attributable to radiative recombination continua, a feature expected from a photoionized gas (Shipley et al. 2015). As noted in that paper, the complete lack of radiative recombination continuua (RRC) features raises the possibility that photoionization is not the dominant process. Kahn et al. (2002) notes that as the electron temperature increases, the width of the RRC increases; an RRC feature present in a collisional plasma is sufficiently broad to essentially disappear into the background. Future observations with higher spectral resolutions will be necessary to determine the degree of photoionization present in CVs.

\section{CONCLUSIONS}

The available grating spectra of $\mathrm{CVs}$ provide a starting point for direct measurements of temperatures and densities in these objects independent of global models. They provide an alternative path to check the models and the physics included in them.

We confirm that a broad range of electron and ionization temperatures exists, but we note that a number of CVs exhibit ranges at least a factor of a few lower than implied by continuum fitting. We can unequivocally conclude that single-temperature models do not describe the observed line emission.

Number density estimates are generally hampered by the strong UV radiation fields from the WDs. However, the Fe XXII line ratio implies maximum number densities of $\sim 10^{13.5} \mathrm{~cm}^{-3}$. The presence of any UV contamination of the Fe XXII lines would then imply a lower real number density. That statement should not be construed to imply that all CVs have low number densities; the range for some CVs will be low. Alternatively, and very likely, we may be measuring different spatial regions approximately sorted by density.

Finally, for the best-exposed spectra, the $\mathrm{He} / \mathrm{H}$ versus $G$ ratios for Fe implies that the plasmas are in, or nearly in, ionization equilibrium, while the same analysis for $\mathrm{S}, \mathrm{Mg}$, and $\mathrm{O}$ reveals clear nonequilibrium conditions-at least in several CVs.

Our conclusions necessarily rest on two fundamentals-one "fixable" and one that requires considerably more work. Regarding the "fixable" component: time allocation committees must take exposure time requests seriously. The rock-bottom problem with the HETG data sets is the relative under exposure of many of the data sets. This problem can be fixed in either of two ways: (1) re-observe the current data using the HETG for considerably longer times to bring the entire data set up to the signal/noise of the $0.5 \mathrm{Ms}$ observation of EX Hya; or (2) use the calorimeter on Astro $H$ to resolve line complexes more cleanly. This second route is the more likely to be taken, yielding as it does higher resolution spectra. We warn the interested reader to be certain to propose for sufficient time to obtain good signal/noise.

Ultimately, our conclusions also rest on the degree to which the observed CVs in this study are representative of their subclass or class - the second fundamental mentioned above, and one that requires more work. We do not detect any clear signal that permits separation of the classes even when using the large "bins" of magnetic versus nonmagnetic. On the basis of the line emission, we can not separate the observed CVs into a "cooling 
flow" group and a "photoionized" group. This does not challenge nor overturn the conclusions of Mukai et al. (2003) because the line emission need not follow the continuum emission. Our inference does make the X-ray emission from CVs more complicated and, potentially, тисh more complicated. The only route out of that box is the accumulation of a sufficient number of high-quality spectra that patterns may be inferred from the line emission, the "more work" mentioned above.

We look forward to the next development in the X-ray spectroscopic study of CVs, namely, the use of a calorimeter to provide 5-10 times better spectral resolution to investigate very detailed line emission processes (e.g., Compton "shoulders," dielectronic scattering). We expect the higher spectral resolution to lead to improved statistics and firm inferences for each $\mathrm{CV}$ - and from there to the population of CVs.

We thank the referee for comments that improved the quality of this paper. E.M.S. acknowledges support from the Chandra X-ray Center through archival grant AR9-0005X to UTSA and partial support from the Vaughan Family endowment.

\section{REFERENCES}

Aizu, K. 1973, PThPh, 49, 1184

Arnaud, M., \& Raymond, J. 1992, ApJ, 398, 394

Bautista, M. A., \& Kallman, T. R. 2000, ApJ, 544, 581

Behar, E., Cottam, J., \& Kahn, S. M. 2001, ApJ, 548, 966

Bernitt, S., Brown, G. V., Rudolph, J. K., et al. 2012, Natur, 492, 225

Blumenthal, G. R., Drake, G. W. F., \& Tucker, W. H. 1972, ApJ, 172, 205

Brown, G. V., Beiersdorfer, P., Chen, H., Chen, M. H., \& Reed, K. J. 2001, ApJL, 557, L75

Canizares, C. R., Davis, J. E., Dewey, D., et al. 2005, PASP, 117, 1144

Canizares, C. R., Huenemoerder, D. P., Davis, D. S., et al. 2000, ApJL, 539, L41

Cassinelli, J. P., Miller, N. A., Waldron, W. L., Macfarlane, J. J., \& Cohen, D. H. 2001, ApJL, 554, L55

Chen, G.-X., \& Pradhan, A. 2005, arXiv:astro.ph.0510534

Cropper, M. 1986, MNRAS, 222, 225

Cropper, M. 1990, SSRv, 54, 195

Doron, R., \& Behar, E. 2002, ApJ, 574, 518

Ezuka, H., \& Ishida, M. 1999, ApJS, 120, 277

Frank, J., King, A., \& Raine, D. 2002, Accretion Power in Astrophysics (3rd ed.; Cambridge: Cambridge Univ. Press)

Gabriel, A. H. 1972, MNRAS, 160, 99

Gabriel, A. H., \& Jordan, C. 1969, MNRAS, 145, 124

Gabriel, A. H., \& Phillips, K. J. H. 1979, MNRAS, 189, 319

Gillaspy, J. D., Lin, T., Tedesco, L., et al. 2011, ApJ, 728, 132
Godet, O., Collin, S., \& Dumont, A.-M. 2004, A\&A, 426, 767

Honeycutt, R. K., Schlegel, E. M., \& Kaitchuck, R. H. 1986, ApJ, 302, 388

Hoogerwerf, R., Brickhouse, N. S., \& Mauche, C. W. 2005, ApJ, 628, 496

Hurwitz, M., Sirk, M., Bowyer, S., \& Ko, Y.-K. 1997, ApJ, 477, 390

Ishida, M., Mukai, K., \& Osborne, J. P. 1994, PASJ, 46, L81

Itoh, K., Ishida, M., \& Kunieda, H. 2006, ApJ, 639, 397

Jimenez-Garate, M. A., Raymond, J. C., Liedahl, D. A., \& Hailey, C. J. 2005, ApJ, 625, 931

Kahn, S., Behar, E., Kinkhabwala, A., \& Savin, D. W. 2002, RSPTA, 360, 1923

Kahn, S., Leutenegger, M. A., Cottam, J., et al. 2001, A\&A, 365, L277

Kallman, T. 1999, Astrophysics Source Code Library, record, 9910.008

Landi, E., \& Phillips, K. J. H. 2005, ApJS, 160, 286

Leutenegger, M. A., Paerels, F. B. S., Kahn, S. M., \& Cohen, D. H. 2006, ApJ, 650, 1096

Liang, G. Y., \& Zhao, G. 2008, AJ, 135, 2291

Liedahl, D. A. 1999, in X-ray Spectroscopy in Astrophysics, ed. J. van Paradijs \& J. A. Bleeker (Dordrecht: Springer), 189

Liedahl, D. A., Kahn, S. M., Osterheld, A. L., \& Goldstein, W. H. 1992, ApJ, 391, 306

Lum, K. S. K., Canizares, C. R., Clark, G. W., et al. 1992, ApJS, 78, 423

Mauche, C. W., Liedahl, D. A., \& Fournier, K. B. 2001, ApJ, 560, 992

Mauche, C. W., Liedahl, D. A., \& Fournier, K. B. 2003a, ApJL, 588, L101

Mauche, C. W., Liedahl, D. A., \& Fournier, K. B. 2005, in AIP Conf No. 774, X-ray Diagnostics of Astrophysical Plasmas, ed. R. K. Smith (Melville, NY: AIP), 133

Mauche, C. W., Liedahl, D. A., Long, K. S., et al. 2003b, BAAS, 35, 631

Mewe, R., Gronenschild, E. H. B. M., \& van den Oord, G. H. J. 1985, A\&AS, 62,197

Mukai, K., Kinkhabwala, A., Peterson, J. R., Kahn, S. M., \& Paerels, F. 2003, ApJL, 586, L77

Perna, R., McDowell, J., Menou, K., Raymond, J., \& Medvedev, M. V. 2003, ApJ, 598, 545

Porquet, D., \& Dubau, J. 2000, A\&AS, 143, 495

Porquet, D., Dubau, J., \& Grosso, N. 2010, SSRv, 157, 103

Porquet, D., Mewe, R., Dubau, J., Raassen, A. J. J., \& Kaastra, J. S. 2001, A\&A, 376, 1113

Pradhan, A. K., \& Shull, J. M. 1981, ApJ, 249, 821

Raasen, A. J. J. 2005, PhS, T119, 13

Rosen, S. R., Mason, K. O., \& Córdova, F. A. 1988, MNRAS, 231, 549

Shipley, H. V., Schlegel, E. M., Rana, V. R., Barrett, P. E., \& Singh, K. P. 2015, ApJS, submitted (Paper I)

Sivia, D. 2006, Data Analysis: A Bayesian Tutorial (2nd ed.; Cambridge: Cambridge Univ. Press)

Smith, R. K., Brickhouse, N. S., Liedahl, D. A., \& Raymond, J. C. 2001, ApJL, 556, L91

Szkody, P., Gänsicke, B. T., Harrison, T. E., et al. 2007, in ASP Conf. Ser. 372, 15th European Workshop on White Dwarfs, ed. R. Napiwotzki \& M. R. Burleigh (San Francisco, CA: ASP), 547

Venter, L. A., \& Meintjes, P. J. 2006, MNRAS, 366, 557

Vrielmann, S., Ness, J.-U., \& Schmitt, J. H. M. M. 2005, A\&A, 439, 287

Waldron, W. L., \& Cassinelli, J. P. 2000, ApJL, 548, L45

Warner, B. 1995, Cataclysmic Variables (Cambridge: Cambridge Univ. Press) 